\title{
Modified Pillai's trace statistics for two high-dimensional sample covariance matrices
}

\author{
Qiuyan Zhang, Jiang Hu*, Zhidong Bai \\ Key Laboratory for Applied Statistics of the Ministry of Education, School of Mathematics and Statistics, Northeast Normal \\ University, China.
}

\begin{abstract}
The goal of this study was to test the equality of two covariance matrices by using modified Pillai's trace statistics under a high-dimensional framework, i.e., the dimension and sample sizes go to infinity proportionally. In this paper, we introduce two modified Pillai's trace statistics and obtain their asymptotic distributions under the null hypothesis. The benefits of the proposed statistics include the following: (1) the sample size can be smaller than the dimensions; (2) the limiting distributions of the proposed statistics are universal; and (3) we do not restrict the structure of the population covariance matrices. The theoretical results are established under mild and practical assumptions, and their properties are demonstrated numerically by simulations and a real data analysis.

Keywords: High-dimensional test, Pillai's trace statistics, Beta matrices, LSS, CLT

2010 MSC: $62 \mathrm{H} 15,62 \mathrm{H} 10$
\end{abstract}

\section{Introduction}

High-dimensional data are common in modern scientific domains, such as finance and wireless communication. Hence, the testing of covariance matrices under high-dimensional settings constitutes an important issue in these areas. The following three main tests have been investigated widely by statisticians 5 for one-sample tests: (i) sphericity test, (ii) identity matrix test, and (iii) diagonal matrix test. Ledoit and Wolf [16] investigated the properties of the sphericity and identity matrix tests when the sample size and the dimension converge to infinity proportionally, and Birke and Dette [7] generalized Ledoit and Wolf's [16] conclusion to the case where the sample size and dimension are not of the same order. Srivastava [25] proved the asymptotic null and alternative distributions of the testing statistics for normally distributed data. Furthermore, Chen et al. [11] proposed a nonparametric method and reported that its data could come from any distribution with a specified data structure. Cai and Ma [8] developed an identity matrix test procedure based on minimax analysis and showed that the power of their test uniformly dominates the power of the corrected likelihood ratio test by Bai et al. [3] over the entire asymptotic regime. Under the

\footnotetext{
* Corresponding author.

Email addresses: zhangqy919@nenu . edu.cn (Qiuyan Zhang), huj156@ nenu . edu . cn (Jiang Hu*), baizd@ nenu. edu. cn (Zhidong Bai)
} 
alternative hypothesis, Chen and Jiang [10] demonstrated the central limit theorem (CLT) of the likelihood ratio test (LRT) statistic. Schott [23], Fisher et al. [12], Srivastava et al. [28], Qiu and Chen [22] and Wu and $\mathrm{Li}$ [29] also analyzed this issue in depth.

Moreover, testing procedures for the equivalence of high-dimensional two-sample covariance matrices are also frequently considered. Regarding the hypothesis test problem,

$$
H_{0}: \Sigma_{1}=\Sigma_{2} \quad \text { v.s. } \quad H_{1}: \Sigma_{1} \neq \Sigma_{2}
$$

where $\Sigma_{1}$ and $\Sigma_{2}$ are two population covariance matrices. Shott [24] proposed a statistic based on the idea of an unbiased estimation of the squared Frobenius norm of $\Sigma_{1}-\Sigma_{2}$, and showed its asymptotic distribution under the condition that the sample sizes and the dimension converge to infinity proportionally. A similar idea was adopted by $\mathrm{Li}$ and $\mathrm{Chen}$ [17] and Gao et al. [13]. In addition, Srivastava [26] considered the lower bound of this Frobenius norm, and Zhang et al. [30] generalized Li and Chen's statistic to multiple samples. Srivastava and Yanagihara [27] considered the distance measure $\operatorname{tr} \Sigma_{1}^{2} /\left(\operatorname{tr} \Sigma_{1}\right)^{2}-\operatorname{tr} \Sigma_{2}^{2} /\left(\operatorname{tr} \Sigma_{2}\right)^{2}$ and proposed a test based on a consistent estimation of this distance. Moreover, Cai et al. [9] developed an estimator to find the maximum difference between entries in two-sample covariance matrices. Bai et 25 al. [3], Zhang et al. [31] and Jiang et al. [14] presented the asymptotic distribution of the correctional LRT under high-dimensional assumptions. Later, Zheng et al. [33] extended the results of Bai et al. [3] to general populations with unknown means.

The goal of this study is to test the hypothesis (1). Assume that our samples $\left\{z_{1}^{(l)}, z_{2}^{(l)}, \ldots, z_{n_{l}}^{(l)}, l=\right.$ $1,2\}$ are drawn independently from populations $z^{(l)}$ with mean $\mu_{l}$ and covariance matrices $\Sigma_{l}$. We denote $\mathbf{S}_{l}^{z}:=\frac{1}{n_{l}-1} \sum_{j=1}^{n_{l}}\left(z_{j}^{(l)}-\bar{z}^{(l)}\right)\left(z_{j}^{(l)}-\bar{z}^{(l)}\right)^{\prime}$, where $\bar{z}^{(l)}=\frac{1}{n_{l}} \sum_{j=1}^{n_{l}} z_{j}^{(l)}$. For the test problem (1), we choose Pillai's classic trace statistic

$$
\operatorname{tr}_{1}^{z}\left(\mathbf{S}_{1}^{z}+\frac{n_{2}}{n_{1}} \mathbf{S}_{2}^{z}\right)^{-1},
$$

which was first proposed by Pillai [21]. For convenience, we subsequently denote $\mathbf{B}_{n}^{z}\left(\mathbf{Z}_{1}, \mathbf{Z}_{2}\right):=\mathbf{S}_{1}^{z}\left(\mathbf{S}_{1}^{z}+\right.$ $\left.\frac{n_{2}}{n_{1}} \mathbf{S}_{2}^{z}\right)^{-1}$, which is called the Beta matrix and was proposed by Bai et al. [5]. From this definition, we note that to guarantee the reversibility of $\mathbf{S}_{1}^{z}+\frac{n_{2}}{n_{1}} \mathbf{S}_{2}^{z}, p$ must be smaller than $n_{1}+n_{2}$. The asymptotic property of Pillai's statistic has been obtained by using the moment method under the condition that the sample sizes diverge but the dimension is fixed. Motivated by Bai et al. [5], in this paper, we modify Pillai's trace statistic by removing the one and zero eigenvalues of $\mathbf{B}_{n}^{z}$, that is,

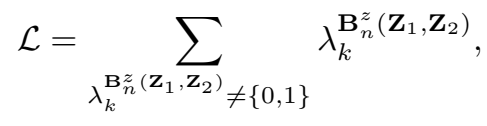

where $\lambda_{k}^{\mathbf{B}_{n}^{z}\left(\mathbf{Z}_{1}, \mathbf{Z}_{2}\right)}$ are eigenvalues of $\mathbf{B}_{n}^{z}\left(\mathbf{Z}_{1}, \mathbf{Z}_{2}\right)$.

In a similar fashion, we modify another of Pillai's trace statistics,

$$
\widetilde{\mathbf{L}}=c_{n_{1}} \operatorname{tr}\left(\frac{1}{c_{n_{1}}} \mathbf{B}_{n}^{z}\left(\mathbf{Z}_{1}, \mathbf{Z}_{2}\right)-\mathbf{I}_{p}\right)^{2}+c_{n_{2}} \operatorname{tr}\left(\frac{1}{c_{n_{2}}} \mathbf{B}_{n}^{z}\left(\mathbf{Z}_{2}, \mathbf{Z}_{1}\right)-\mathbf{I}_{p}\right)^{2}
$$


and transform $\widetilde{\mathbf{L}}$ to

$$
\widetilde{\mathcal{L}}=\sum_{\lambda_{k}^{\mathbf{B} z\left(\mathbf{z}_{1}, \mathbf{z}_{2}\right)} \neq\{0,1\}} \sum_{\lambda_{k^{\prime}}^{\mathbf{B} z\left(\mathbf{z}_{2}, \mathbf{z}_{1}\right)} \neq\{0,1\}}\left[c_{n_{1}}\left(\frac{1}{c_{n_{1}}} \lambda_{k}^{\mathbf{B}_{n}^{z}\left(\mathbf{z}_{1}, \mathbf{Z}_{2}\right)}-1\right)^{2}+c_{n_{2}}\left(\frac{1}{c_{n_{2}}} \lambda_{k^{\prime}}^{\mathbf{B}_{n}^{z}\left(\mathbf{z}_{2}, \mathbf{Z}_{1}\right)}-1\right)^{2}\right],
$$

where $c_{n_{1}}=\frac{n_{1}}{n_{1}+n_{2}}, c_{n_{2}}=\frac{n_{2}}{n_{1}+n_{2}}, \mathbf{B}_{n}^{z}\left(\mathbf{Z}_{2}, \mathbf{Z}_{1}\right)=\mathbf{S}_{2}^{z}\left(\mathbf{S}_{2}^{z}+\frac{n_{1}}{n_{2}} \mathbf{S}_{1}^{z}\right)^{-1}$ and $\lambda_{k^{\prime}}^{\mathbf{B}_{n}^{z}\left(\mathbf{Z}_{2}, \mathbf{Z}_{1}\right)}$ are eigenvalues of $\mathbf{B}_{n}^{z}\left(\mathbf{Z}_{2}, \mathbf{Z}_{1}\right)$. In the next section, we will show the CLTs of $\mathcal{L}$ and $\widetilde{\mathcal{L}}$ under a high-dimensional setting under the null hypothesis.

The main technical tool employed in this paper is random matrix theory (RMT), which is a powerful method when the dimension $p$ is large. Marchenko and Pastur [18] determined the limiting spectral distribution of a large-dimensional sample covariance matrix. Bai and Silverstein [1] proposed a CLT for the linear spectral statistics (LSS) of large-dimensional sample covariance matrices that highlights this issue. Zheng [32] considers a CLT for the LSS of a large-dimensional F matrix, which is used to fulfill the two-sample test. However, the drawback of their method is that the dimension $p$ must be smaller than $\max \left\{n_{1}, n_{2}\right\}$. Bai and Yao [2] focused on the spiked model, which was first proposed by Johnstone [15], and established a limit theorem of extreme sample eigenvalues. Similar works include Baik and Silverstein [6], Paul [20], Bai et al. [4] and Passemier et al. [19]. Recently, Bai et al. [5] proved the CLT for the LSS of the Beta matrix using the asymptotically normally distributed property of the sum of the martingale difference sequence and extended the dimension to a high-dimensional situation.

One should notice that the main technical tool used here is Cauchy's residue theorem-the same technique utilized in Zhang et al. [31]; however, the difference is that the integrands in the current paper are linear functions, whereas the integrands for the LRT statistics proposed in Zhang et al. [31] are logarithmic functions. Moreover, these linear functions can be implemented more rapidly and in a less source-consuming way than the abovementioned logarithmic functions, which have greater computational complexity. In addition, it is clear that when $\frac{p}{n_{1}}$ or $\frac{p}{n_{2}}$ tend to 1 , the Beta matrix will have eigenvalues tend to 0 or 1 which causes logarithm function tend to infinity. Therefore, the variance of the statistic would tend to infinity which surely makes the test less powerful. However, due to the linearity of the integrand functions, Pillai's trace statistics can be utilized in a space where $\frac{p}{n_{1}}=1$ or $\frac{p}{n_{2}}=1$. Therefore, Bai et al. [5], Zhang et al. [31] and the current paper can be viewed as a series of works aimed at improving the classic test statistics of two-sample covariance matrices (see (1.2) in Bai et al. [5]) from a low-dimensional framework to a high-dimensional framework. In Section 3, we compare the test statistics proposed in this paper with Li and Chen's [17] statistic, Cai et al.'s [9] statistic and Zhang et al.'s [31] statistics through simulations.

The remainder of this paper is organized as follows. Section 2 presents the main conclusions related to the proposed statistics. The results of the simulations, including the comparison with $\mathrm{Li}$ and Chen's [17] statistic, Cai et al.'s [9] statistic and Zhang et al.'s [31] statistics, are presented in Section 3. Section 4 60 includes an analysis using real Standard and Poor's 500 index data. The proof is presented in the Appendix. 


\section{Asymptotically normal property}

In this section, we present the main results of this paper. In the sequel, we assume the samples satisfy

$$
z_{i}^{(l)}=\Sigma_{l}^{1 / 2} x_{i}^{(l)}+\mu_{l}
$$

where $x_{i}^{(l)}=\left(x_{i 1}^{(l)}, \ldots, x_{i p}^{(l)}\right)^{\prime}$ and $l=1,2$. Because the trace statistic is invariant under the null hypothesis $\Sigma_{1}=\Sigma_{2}$, we have

$$
\operatorname{tr}_{1}^{z}\left(\mathbf{S}_{1}^{z}+\frac{n_{2}}{n_{1}} \mathbf{S}_{2}^{z}\right)^{-1}=\operatorname{tr}_{1}^{x}\left(\mathbf{S}_{1}^{x}+\frac{n_{2}}{n_{1}} \mathbf{S}_{2}^{x}\right)^{-1}
$$

where $\mathbf{S}_{l}^{x}:=\frac{1}{n_{l}-1} \sum_{j=1}^{n_{l}}\left(x_{j}^{(l)}-\bar{x}^{(l)}\right)\left(x_{j}^{(l)}-\bar{x}^{(l)}\right)^{\prime}$ and $\bar{x}^{(l)}=\frac{1}{n_{l}} \sum_{j=1}^{n_{l}} x_{j}^{(l)}, l=1,2$. Then, we redefine the Beta matrix as $\mathbf{B}_{n}^{x}\left(\mathbf{X}_{1}, \mathbf{X}_{2}\right)=\mathbf{S}_{1}^{x}\left(\mathbf{S}_{1}^{x}+\frac{n_{2}}{n_{1}} \mathbf{S}_{2}^{x}\right)^{-1}, \mathbf{B}_{n}^{x}\left(\mathbf{X}_{2}, \mathbf{X}_{1}\right)=\mathbf{S}_{2}^{x}\left(\mathbf{S}_{2}^{x}+\frac{n_{1}}{n_{2}} \mathbf{S}_{1}^{x}\right)^{-1}$ and transform our statistics into the following forms

$$
\begin{aligned}
& \mathcal{L}=\sum_{\lambda_{k}^{\mathbf{B}_{n}^{x}\left(\mathbf{X}_{1}, \mathbf{X}_{2}\right)} \neq\{0,1\}} \lambda_{k}^{\mathbf{B}_{n}^{x}\left(\mathbf{X}_{1}, \mathbf{X}_{2}\right)}, \\
& \widetilde{\mathcal{L}}=\sum_{\lambda_{k}^{\mathbf{B}_{n}^{x}\left(\mathbf{x}_{1}, \mathbf{X}_{2}\right)} \neq\{0,1\}} \sum_{\lambda_{k^{\prime}}^{\mathbf{B}_{n}^{x}\left(\mathbf{X}_{2}, \mathbf{X}_{1}\right)} \neq\{0,1\}}\left[c_{n_{1}}\left(\frac{1}{c_{n_{1}}} \lambda_{k}^{\mathbf{B}_{n}^{x}\left(\mathbf{X}_{1}, \mathbf{X}_{2}\right)}-1\right)^{2}+c_{n_{2}}\left(\frac{1}{c_{n_{2}}}\left(1-\lambda_{k^{\prime}}^{\mathbf{B}_{n}^{x}\left(\mathbf{X}_{1}, \mathbf{X}_{2}\right)}\right)-1\right)^{2}\right] .
\end{aligned}
$$

Under the following mild assumptions

(1) $\left\{x_{i j}^{(l)}, i=1, \ldots, p, j=1, \ldots, n_{l}\right\}$ are independent and identically distributed real random variables;

(2) As $\min \left\{p, n_{1}, n_{2}\right\} \rightarrow \infty, y_{n_{1}}:=\frac{p}{n_{1}} \rightarrow y_{1} \in(0,+\infty), y_{n_{2}}:=\frac{p}{n_{2}} \rightarrow y_{2} \in(0,+\infty)$ and $\alpha_{n}:=$ $n_{2} / n_{1} \rightarrow \alpha>0$

(3) As $\min \left\{p, n_{1}, n_{2}\right\} \rightarrow \infty, h_{n}:=\sqrt{y_{n_{1}}+y_{n_{2}}-y_{n_{1}} y_{n_{2}}} \rightarrow \sqrt{y_{1}+y_{2}-y_{1} y_{2}}>0$;

(4) $\mathbf{E} x_{i j}^{(l)}=0, \mathbf{E}\left(x_{i j}^{(l)}\right)^{2}=1, \Delta_{1}=: \mathbf{E}\left(x_{i j}^{(1)}\right)^{4}-3<\infty$ and $\Delta_{2}:=\mathbf{E}\left(x_{i j}^{(2)}\right)^{4}-3<\infty$, we draw the following conclusion about the modified Pillai's statistic $T_{1}$.

Theorem 1. Under assumptions (1) - (4), as $\min \left\{p, n_{1}, n_{2}\right\}$ tends to infinity, we have

$$
T_{1}:=\frac{\mathcal{L}-p l_{n}-\mu_{n}}{\nu_{n}} \stackrel{D}{\rightarrow} \mathcal{N}(0,1),
$$

where

$$
l_{n}=\frac{y_{n_{2}}}{y_{n_{1}}+y_{n_{2}}}-\frac{y_{n_{2}}-1}{y_{n_{2}}} \delta_{\left(y_{n_{2}}>1\right)}, \quad \mu_{n}=-\frac{\Delta_{1} y_{n_{1}}^{2} y_{n_{2}}^{2} h_{n}^{2}}{\left(y_{n_{1}}+y_{n_{2}}\right)^{4}}-\frac{\Delta_{2} y_{n_{1}}^{2} y_{n_{2}}^{2} h_{n}^{2}}{\left(y_{n_{1}}+y_{n_{2}}\right)^{4}}
$$

and

$$
\nu_{n}^{2}=\frac{2 y_{n_{1}}^{2} y_{n_{2}}^{2} h_{n}^{2}}{\left(y_{n_{1}}+y_{n_{2}}\right)^{4}}+\frac{\left(y_{n_{1}} \Delta_{1}+y_{n_{2}} \Delta_{2}\right) y_{n_{1}}^{2} y_{n_{2}}^{2} h_{n}^{4}}{\left(y_{n_{1}}+y_{n_{2}}\right)^{6}} .
$$

Here, $\delta_{(\cdot)}$ denotes the indicator function and $\stackrel{D}{\rightarrow}$ denotes convergence in distribution.

70 The proof of this theorem is in the Appendix. The following theorem is based on $\widetilde{\mathcal{L}}$. 
Theorem 2. Under assumptions (1) - (4), as $\min \left\{p, n_{1}, n_{2}\right\} \rightarrow \infty$, we have

$$
T_{2}:=\frac{\tilde{\mathcal{L}}-p \tilde{l}_{n}-\tilde{\mu}_{n}}{\tilde{\nu}_{n}} \stackrel{D}{\rightarrow} \mathcal{N}(0,1),
$$

where

$$
\begin{gathered}
\tilde{l}_{n}=\frac{y_{n_{1}} y_{n_{2}}}{y_{n_{1}}+y_{n_{2}}}+\frac{\left(1-y_{n_{1}}\right) y_{n_{2}}}{y_{n_{1}}^{2}} \delta_{\left(y_{n_{1}}>1\right)}+\frac{y_{n_{1}}\left(1-y_{n_{2}}\right)}{y_{n_{2}}^{2}} \delta_{\left(y_{n_{2}}>1\right)}, \\
\text { and } \tilde{\mu}_{n}=\frac{y_{n_{1}} y_{n_{2}} h_{n}^{2}}{\left(y_{n_{1}}+y_{n_{2}}\right)^{2}}+\frac{\Delta_{1} y_{n_{1}}^{2} y_{n_{2}} h_{n}^{2}\left(h_{n}^{2}+2 y_{n_{2}}\left(y_{n_{2}}-y_{n_{1}}\right)\right)}{\left(y_{n_{1}}+y_{n_{2}}\right)^{4}}+\frac{\Delta_{2} y_{n_{2}}^{2} y_{n_{1}} h_{n}^{2}\left(h_{n}^{2}+2 y_{n_{1}}\left(y_{n_{1}}-y_{n_{2}}\right)\right)}{\left(y_{n_{1}}+y_{n_{2}}\right)^{4}}, \\
\tilde{\nu}_{n}^{2}=\frac{4 y_{n_{1}}^{2} y_{n_{2}}^{2} h_{n}^{2}\left(h_{n}^{2}+2\left(y_{n_{1}}-y_{n_{2}}\right)^{2}\right)}{\left(y_{n_{1}}+y_{n_{2}}\right)^{4}}+\frac{\left(y_{n_{1}} \Delta_{1}+y_{n_{2}} \Delta_{2}\right) 4 y_{n_{1}}^{2} y_{n_{2}}^{2} h_{n}^{4}\left(y_{n_{1}}-y_{n_{2}}\right)^{2}}{\left(y_{n_{1}}+y_{n_{2}}\right)^{6}}
\end{gathered}
$$

The proof of this theorem is also in the Appendix.

Remark 1. In contrast to the LRT statistics in Zhang et al. [31], the modified Pillai's trace statistics $T_{1}$ and $T_{2}$ proposed in this paper are feasible when $y_{1}=1$ or $y_{2}=1$, because all the limits, means and variances of the modified Pillai's trace statistics are continuous in their definitional domain. We can also find from

75 Figure 1 in Zhang et al. [31] that when $y_{1}$ or $y_{2}$ are close to 1 , the mean and variance of the LRT statistics increase rapidly, resulting in poor power. For illustration purposes, three-dimensional shaded figures of $\tilde{\mu}_{n}$ and $\tilde{\nu}_{n}^{2}$ with $\Delta_{1}=\Delta_{2}=0$ are shown in Figure 1 and Figure 2 , respectively.
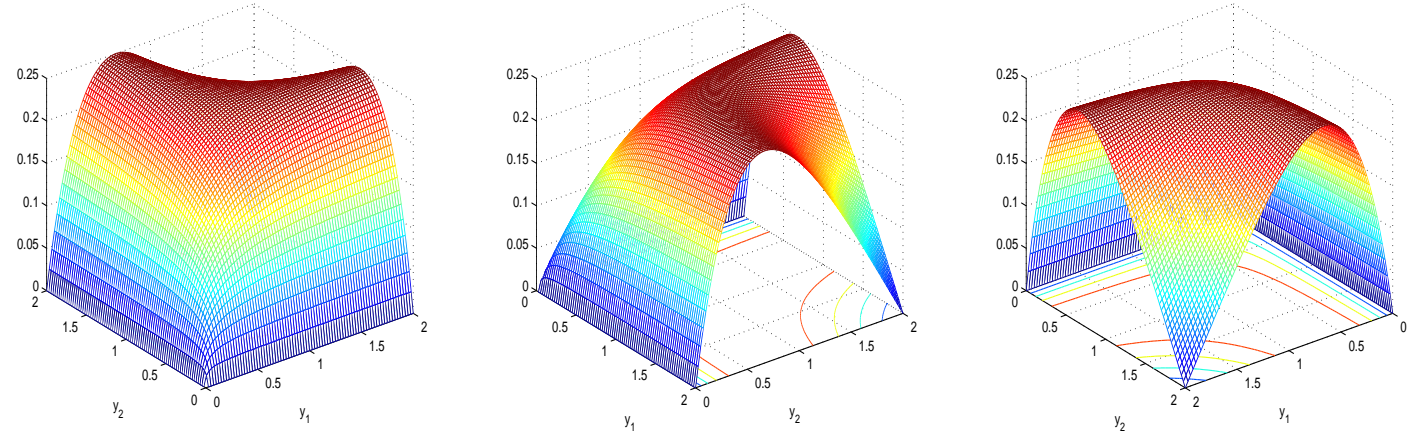

Figure 1: Three-dimensional shaded figure of $\tilde{\mu}_{n}$ from three angles when $y_{1} \in(0,2)$ and $y_{2} \in(0,2)$.

Remark 2. When $\Delta_{l} \neq 0$, Zhang et al. [31] provided the following estimators

$$
\begin{aligned}
\hat{\Delta}_{1}= & (1-y)^{2} \frac{\sum_{j=1}^{n_{1}}\left[\left(z_{j}^{(1)}-\bar{z}^{(1)}\right)^{\prime}\left(c_{11} \mathbf{S}_{1 j}^{z}+c_{12} \mathbf{S}_{2}^{z}\right)^{-1}\left(z_{j}^{(1)}-\bar{z}^{(1)}\right)-\frac{p}{1-y}\right]^{2}}{p n_{1}} \\
& -\frac{2}{1-y} \\
\hat{\Delta}_{2}= & (1-y)^{2} \frac{\sum_{j=1}^{n_{2}}\left[\left(z_{j}^{(2)}-\bar{z}^{(2)}\right)^{\prime}\left(c_{21} \mathbf{S}_{1}^{z}+c_{22} \mathbf{S}_{2 j}^{z}\right)^{-1}\left(z_{j}^{(2)}-\bar{z}^{(2)}\right)-\frac{p}{1-y}\right]^{2}}{p n_{2}} \\
& -\frac{2}{1-y}
\end{aligned}
$$



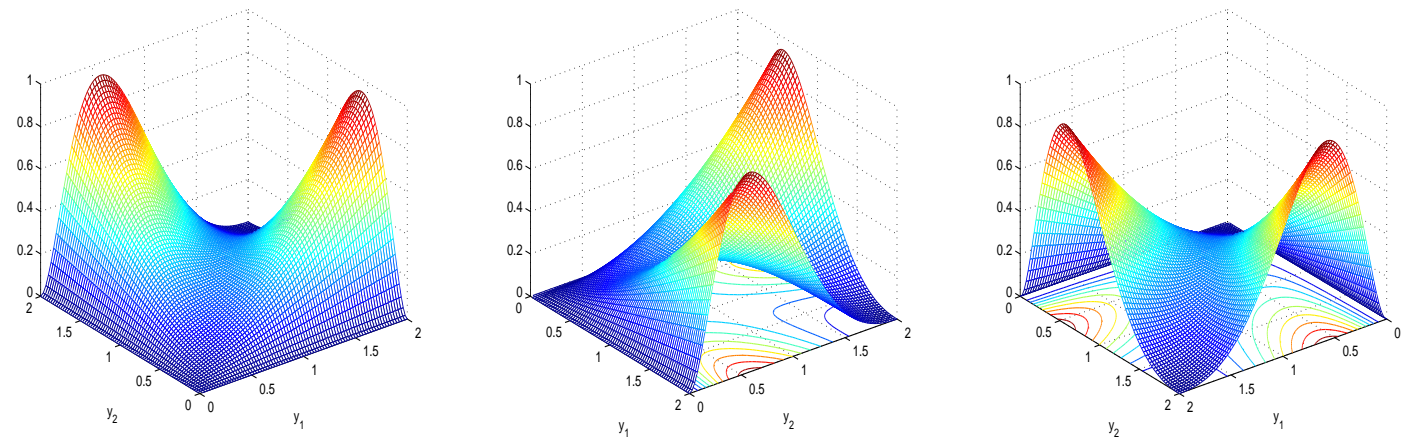

Figure 2: Three-dimensional shaded figure of $\tilde{\nu}_{n}^{2}$ from three angles when $y_{1} \in(0,2)$ and $y_{2} \in(0,2)$.

where $y=\frac{p}{n_{1}+n_{2}-1}, c_{11}=\frac{n_{1}-1}{n_{1}+n_{2}-1}, c_{12}=\frac{n_{2}}{n_{1}+n_{2}-1}, c_{21}=\frac{n_{1}}{n_{1}+n_{2}-1}, c_{22}=\frac{n_{2}-1}{n_{1}+n_{2}-1}$ and $\mathbf{S}_{l j}^{z}$ is the sample covariance matrix by removing the vector $z_{j}^{(l)}$ from the $l$-th sample, $l=1,2$; Zhang et al. also showed that these estimators are weakly consistent and asymptotically unbiased under the null hypothesis. Under the alternative hypothesis, when $p<n_{1}$ and $p<n_{2}$, the estimators are also applicable after applying a small modification. However, if the linear model setting (2) does not hold or if $p \geq n_{1}+n_{2}$, then to the best of our knowledge, no consistent estimator of $\Delta_{i}$ exists.

\section{Simulation}

In this section, we compare the modified Pillai's trace statistics $T_{1}$ and $T_{2}$ with four other statistics: $T_{l c}$, $T_{c l x}, T_{z h b}^{1}$ and $T_{z h b}^{2}$ proposed by $\mathrm{Li}$ and Chen [17], Cai et al. [9] and Zhang et al. [31], respectively. In the first subsection, we compare the empirical sizes and powers of the proposed statistics $T_{1}$ and $T_{2}$ with $T_{l c}, T_{c l x}, T_{z h b}^{1}$ and $T_{z h b}^{2}$ in some different settings. Because the properties of $T_{1}$ and $T_{2}$ are universal and invariant under the null hypothesis $\Sigma_{1}=\Sigma_{2}$, and their powers depend only on the eigenvalues of $\Sigma_{1} \Sigma_{2}^{-1}$, we organize four different targeted models and two different distributions. In the second subsection, we use the Jarque-Bera (J-B) and Kolmogorov-Smirnov (K-S) tests to illustrate how well the proposed statistics fit their limiting distribution with a finite sample.

\subsection{Comparisons of empirical sizes and powers}

First, we consider a comparison with $T_{l c}$ and $T_{c l x}$. To test hypothesis 11 , we randomly generate $x_{j}^{(l)}$ from a standard multivariate normal distribution $N\left(0_{p}, I_{p}\right)$, and let $z_{j}^{(l)}=\Sigma_{l}^{1 / 2} x_{j}^{(l)}$. Simultaneously, to realize the empirical size and power of the test, we define

$$
\Sigma_{2}=\left(1+\frac{\delta}{n_{1}}\right) \Sigma_{1}
$$

When $\delta=0$, we achieve the empirical size. $\Sigma_{1}$ follows the following four models:

${ }_{95} \quad$ Model 1: $\Sigma_{1}=I_{p}$; 
Model 2: $\Sigma_{1}=\operatorname{Diag}\left(p^{2}, 1, \ldots, 1\right)$;

Model 3: $\Sigma_{1}=D^{1 / 2} \Sigma^{*} D^{1 / 2}$, where $D=\operatorname{Diag}\left(d_{1}, d_{2}, \ldots, d_{p}\right), d_{i}=\operatorname{Unif}(0.5,2.5)$ and $\Sigma^{*}=\left(\sigma_{i j}^{*}\right)$, $\sigma_{i i}^{*}=1, \sigma_{i j}^{*}=0.5$ for $5(k-1)+1 \leq i \neq j \leq 5 k, k=1, \ldots,\lceil p / 5\rceil$; otherwise, $\sigma_{i j}^{*}=0$, $i, j=1,2, \ldots, p$;

Model 4: $\Sigma_{1}=\left(0.5 I_{p}+0.5 \mathbf{1}_{p} \mathbf{1}_{p}^{\prime}\right)$, where $\mathbf{1}_{p}$ is an all-ones vector.

We set the actual size to $5 \%$ for 1,000 repetitive simulations. The sample sizes $\left(n_{1}, n_{2}\right)$ increase from $(25,35)$ to $(400,560)$. To fulfill all the conditions, $p$ is selected under the assumption that $p<\min \left\{n_{1}, n_{2}\right\}$, $\min \left\{n_{1}, n_{2}\right\}<p<\max \left\{n_{1}, n_{2}\right\}$ or $p>\max \left\{n_{1}, n_{2}\right\}$. The $\Sigma_{l}$ in Model 1 comes from Li and Chen [17] and satisfies the corresponding assumption that $\Sigma_{l}$ has a moderate eigenvalue. However, Model 2 fails to satisfy this demand. Model 3 comes from Cai et al. [9] and is quite sparse. Because $T_{c l x}$ is established on the corresponding elements of the two covariance matrices and requires a sparsity condition, for comparison, we choose the Model 4, which is unable to satisfy the sparse condition. The simulation results for Models 1-4 are reported in Tables 114, respectively.

\begin{tabular}{|c|c|c|c|c|c|c|c|c|c|c|}
\hline \multirow[t]{2}{*}{$\left(n_{1}, n_{2}\right)$} & \multirow{2}{*}{$\begin{array}{c}\text { Method } \\
\mathrm{p}\end{array}$} & \multicolumn{3}{|c|}{ Size $(\delta=0)$} & \multicolumn{3}{|c|}{ Power $(\delta=5)$} & \multicolumn{3}{|c|}{ Power $(\delta=10)$} \\
\hline & & 20 & 30 & 40 & 20 & 30 & 40 & 20 & 30 & 40 \\
\hline \multirow{3}{*}{$(25,35)$} & $T_{1}$ & 0.051 & 0.055 & 0.047 & 0.948 & 0.963 & 0.943 & 1 & 1 & 1 \\
\hline & $T_{2}$ & 0.046 & 0.052 & 0.042 & 0.325 & 0.436 & 0.607 & 0.860 & 0.952 & 0.999 \\
\hline & $T_{l c}$ & 0.064 & 0.070 & 0.061 & 0.220 & 0.205 & 0.207 & 0.770 & 0.818 & 0.806 \\
\hline \multirow{4}{*}{$(50,70)$} & $\mathrm{p}$ & 40 & 60 & 80 & 40 & 60 & 80 & 40 & 60 & 80 \\
\hline & $T_{1}$ & 0.049 & 0.051 & 0.048 & 0.955 & 0.964 & 0.959 & 1 & 1 & 1 \\
\hline & $T_{2}$ & 0.044 & 0.054 & 0.042 & 0.260 & 0.431 & 0.602 & 0.832 & 0.963 & 0.994 \\
\hline & $T_{l c}$ & 0.062 & 0.053 & 0.073 & 0.099 & 0.111 & 0.112 & 0.516 & 0.471 & 0.501 \\
\hline \multirow{4}{*}{$(100,140)$} & $\mathrm{p}$ & 80 & 120 & 160 & 80 & 120 & 160 & 80 & 120 & 160 \\
\hline & $T_{1}$ & 0.047 & 0.046 & 0.063 & 0.973 & 0.982 & 0.971 & 1 & 1 & 1 \\
\hline & $T_{2}$ & 0.045 & 0.055 & 0.054 & 0.256 & 0.434 & 0.605 & 0.768 & 0.965 & 0.992 \\
\hline & $T_{l c}$ & 0.059 & 0.062 & 0.051 & 0.069 & 0.071 & 0.059 & 0.216 & 0.208 & 0.215 \\
\hline \multirow{4}{*}{$(200,280)$} & $\mathrm{p}$ & 160 & 240 & 320 & 160 & 240 & 320 & 160 & 240 & 320 \\
\hline & $T_{1}$ & 0.043 & 0.055 & 0.049 & 0.973 & 0.983 & 0.978 & 1 & 1 & 1 \\
\hline & $T_{2}$ & 0.052 & 0.044 & 0.049 & 0.240 & 0.417 & 0.600 & 0.756 & 0.950 & 0.991 \\
\hline & $T_{l c}$ & 0.055 & 0.048 & 0.064 & 0.054 & 0.070 & 0.058 & 0.098 & 0.088 & 0.102 \\
\hline \multirow{4}{*}{$(400,560)$} & $\mathrm{p}$ & 320 & 480 & 640 & 320 & 480 & 640 & 320 & 480 & 640 \\
\hline & $T_{1}$ & 0.056 & 0.054 & 0.045 & 0.971 & 0.981 & 0.974 & 1 & 1 & 1 \\
\hline & $T_{2}$ & 0.046 & 0.051 & 0.051 & 0.253 & 0.434 & 0.566 & 0.715 & 0.959 & 0.990 \\
\hline & $T_{l c}$ & 0.059 & 0.053 & 0.049 & 0.052 & 0.047 & 0.051 & 0.048 & 0.061 & 0.052 \\
\hline
\end{tabular}

Table 1: Empirical size and power from 1,000 repeated simulations comparing $T_{1}, T_{2}$ and $T_{l c}$ based on Model 1 under the normal assumption.

Tables 14 show that when $\min \left\{n_{1}, n_{2}, p\right\}$ is large, the modified Pillai's trace statistics have relatively good sizes, and in terms of $\delta$, the modified Pillai's trace statistics are more efficient than are $T_{l c}$ and $T_{c l x}$. 


\begin{tabular}{|c|c|c|c|c|c|c|c|c|c|c|}
\hline \multirow[t]{2}{*}{$\left(n_{1}, n_{2}\right)$} & \multirow{2}{*}{$\frac{\text { Method }}{\mathrm{p}}$} & \multicolumn{3}{|c|}{ Size $(\delta=0)$} & \multicolumn{3}{|c|}{ Power $(\delta=5)$} & \multicolumn{3}{|c|}{ Power $(\delta=10)$} \\
\hline & & 20 & 30 & 40 & 20 & 30 & 40 & 20 & 30 & 40 \\
\hline \multirow{3}{*}{$(25,35)$} & $T_{1}$ & 0.048 & 0.038 & 0.046 & 0.949 & 0.966 & 0.948 & 1 & 1 & 1 \\
\hline & $T_{2}$ & 0.045 & 0.043 & 0.036 & 0.323 & 0.439 & 0.614 & 0.853 & 0.969 & 0.992 \\
\hline & $T_{l c}$ & 0.091 & 0.086 & 0.093 & 0.205 & 0.226 & 0.216 & 0.477 & 0.483 & 0.514 \\
\hline \multirow{4}{*}{$(50,70)$} & $\mathrm{p}$ & 40 & 60 & 80 & 40 & 60 & 80 & 40 & 60 & 80 \\
\hline & $T_{1}$ & 0.040 & 0.065 & 0.045 & 0.956 & 0.966 & 0.960 & 1 & 1 & 1 \\
\hline & $T_{2}$ & 0.056 & 0.059 & 0.050 & 0.257 & 0.433 & 0.622 & 0.825 & 0.956 & 0.993 \\
\hline & $T_{l c}$ & 0.074 & 0.097 & 0.077 & 0.167 & 0.166 & 0.166 & 0.345 & 0.385 & 0.374 \\
\hline \multirow{4}{*}{$(100,140)$} & $\mathrm{p}$ & 80 & 120 & 160 & 80 & 120 & 160 & 80 & 120 & 160 \\
\hline & $T_{1}$ & 0.043 & 0.046 & 0.052 & 0.971 & 0.970 & 0.970 & 1 & 1 & 1 \\
\hline & $T_{2}$ & 0.047 & 0.048 & 0.053 & 0.276 & 0.422 & 0.584 & 0.809 & 0.967 & 0.994 \\
\hline & $T_{l c}$ & 0.092 & 0.100 & 0.076 & 0.111 & 0.114 & 0.119 & 0.240 & 0.253 & 0.263 \\
\hline \multirow{4}{*}{$(200,280)$} & $\mathrm{p}$ & 160 & 240 & 320 & 160 & 240 & 320 & 160 & 240 & 320 \\
\hline & $T_{1}$ & 0.054 & 0.051 & 0.055 & 0.971 & 0.981 & 0.975 & 1 & 1 & 1 \\
\hline & $T_{2}$ & 0.053 & 0.048 & 0.054 & 0.270 & 0.403 & 0.602 & 0.759 & 0.962 & 0.998 \\
\hline & $T_{l c}$ & 0.093 & 0.102 & 0.074 & 0.098 & 0.111 & 0.131 & 0.175 & 0.181 & 0.171 \\
\hline \multirow{4}{*}{$(400,560)$} & $\mathrm{p}$ & 320 & 480 & 640 & 320 & 480 & 640 & 320 & 480 & 640 \\
\hline & $T_{1}$ & 0.051 & 0.037 & 0.038 & 0.979 & 0.989 & 0.981 & 1 & 1 & 1 \\
\hline & $T_{2}$ & 0.052 & 0.046 & 0.044 & 0.250 & 0.417 & 0.599 & 0.719 & 0.961 & 0.997 \\
\hline & $T_{l c}$ & 0.085 & 0.081 & 0.090 & 0.090 & 0.082 & 0.086 & 0.118 & 0.126 & 0.129 \\
\hline
\end{tabular}

Table 2: Empirical size and power from 1,000 repeated simulations comparing $T_{1}, T_{2}$ and $T_{l c}$ based on Model 2 under the normal assumption.

That is, $T_{1}$ and $T_{2}$ can be utilized to distinguish the two different covariance matrices even when $\delta$ is small. To illustrate the outstanding efficiency of the modified Pillai's trace statistics, we show scatter plots in Figures 344 under $\left(n_{1}, n_{2}, p\right)=(50,70,40)$ as $\delta$ increases from 0 to 20 . The modified Pillai's trace statistics tend toward 1 more quickly than do either $T_{l c}$ or $T_{c l x}$.

Because the proposed statistics are also applicable under nonnormal conditions, we also simulate $x_{j}^{(l)}$ with the $p$-dimensional uniform distribution $\operatorname{Unif}_{p}(-\sqrt{3}, \sqrt{3})$; that is, all elements of $x_{j}^{(l)}$ are independently generated from the uniform distribution $\operatorname{Unif}(-\sqrt{3}, \sqrt{3})$. The results of the four models for $\Sigma_{l}$ are presented in Tables 5,8 and in Figures 5 , respectively. From these results, we find that the performances of $T_{1}$ under uniform distribution condition are very similar to its performances under the normal distribution condition, and so is to the performances of $T_{2}$. Therefore, that coincides with our claim that the proposed statistics are universal.

We also compare $T_{1}, T_{2}, T_{z h b}^{1}$ and $T_{z h b}^{2}$ for four models under normal distribution condition in Figures 912. From these simulations, we find that $T_{1}$ seems to be more powerful than the other three statistics under these four models. In addition, because the power of $T_{z h b}^{1}$ and $T_{z h b}^{2}$ also depend only on the eigenvalues 125 of $\Sigma_{1} \Sigma_{2}^{-1}$, the four figures appear quite similar. 


\begin{tabular}{|c|c|c|c|c|c|c|c|c|c|c|}
\hline \multirow[t]{2}{*}{$\left(n_{1}, n_{2}\right)$} & \multirow{2}{*}{$\frac{\text { Method }}{\mathrm{p}}$} & \multicolumn{3}{|c|}{ Size $(\delta=0)$} & \multicolumn{3}{|c|}{ Power $(\delta=5)$} & \multicolumn{3}{|c|}{ Power $(\delta=10)$} \\
\hline & & 20 & 30 & 40 & 20 & 30 & 40 & 20 & 30 & 40 \\
\hline \multirow{3}{*}{$(25,35)$} & $T_{1}$ & 0.051 & 0.054 & 0.055 & 0.951 & 0.964 & 0.940 & 1 & 1 & 1 \\
\hline & $T_{2}$ & 0.046 & 0.043 & 0.048 & 0.337 & 0.423 & 0.623 & 0.871 & 0.964 & 0.995 \\
\hline & $T_{c l x}$ & 0.070 & 0.091 & 0.075 & 0.084 & 0.090 & 0.087 & 0.164 & 0.173 & 0.158 \\
\hline \multirow{4}{*}{$(50,70)$} & $\mathrm{p}$ & 40 & 60 & 80 & 40 & 60 & 80 & 40 & 60 & 80 \\
\hline & $T_{1}$ & 0.051 & 0.045 & 0.056 & 0.964 & 0.971 & 0.967 & 1 & 1 & 1 \\
\hline & $T_{2}$ & 0.054 & 0.042 & 0.043 & 0.296 & 0.431 & 0.615 & 0.832 & 0.975 & 0.995 \\
\hline & $T_{c l x}$ & 0.045 & 0.054 & 0.055 & 0.065 & 0.049 & 0.071 & 0.078 & 0.081 & 0.073 \\
\hline \multirow{4}{*}{$(100,140)$} & $\mathrm{p}$ & 80 & 120 & 160 & 80 & 120 & 160 & 80 & 120 & 160 \\
\hline & $T_{1}$ & 0.052 & 0.050 & 0.049 & 0.973 & 0.978 & 0.970 & 1 & 1 & 1 \\
\hline & $T_{2}$ & 0.053 & 0.042 & 0.064 & 0.281 & 0.422 & 0.599 & 0.805 & 0.954 & 0.994 \\
\hline & $T_{c l x}$ & 0.042 & 0.044 & 0.050 & 0.052 & 0.045 & 0.031 & 0.060 & 0.049 & 0.055 \\
\hline \multirow{4}{*}{$(200,280)$} & $\mathrm{p}$ & 160 & 240 & 320 & 160 & 240 & 320 & 160 & 240 & 320 \\
\hline & $T_{1}$ & 0.054 & 0.039 & 0.048 & 0.971 & 0.977 & 0.976 & 1 & 1 & 1 \\
\hline & $T_{2}$ & 0.060 & 0.036 & 0.048 & 0.258 & 0.457 & 0.625 & 0.762 & 0.957 & 0.993 \\
\hline & $T_{c l x}$ & 0.050 & 0.032 & 0.043 & 0.045 & 0.046 & 0.043 & 0.050 & 0.052 & 0.044 \\
\hline \multirow{4}{*}{$(400,560)$} & $\mathrm{p}$ & 320 & 480 & 640 & 320 & 480 & 640 & 320 & 480 & 640 \\
\hline & $T_{1}$ & 0.046 & 0.047 & 0.040 & 0.966 & 0.987 & 0.971 & 1 & 1 & 1 \\
\hline & $T_{2}$ & 0.054 & 0.046 & 0.038 & 0.254 & 0.423 & 0.621 & 0.743 & 0.950 & 0.994 \\
\hline & $T_{c l x}$ & 0.044 & 0.046 & 0.044 & 0.038 & 0.044 & 0.036 & 0.045 & 0.054 & 0.050 \\
\hline
\end{tabular}

Table 3: Empirical size and power from 1,000 repeated simulations comparing $T_{1}, T_{2}$ and $T_{c l x}$ based on Model 3 under the normal assumption.

\subsection{Goodness-of-fit tests}

In this subsection, we compare the goodness-of-fit tests of the proposed statistics with those of $T_{z h b}^{1}$, $T_{z h b}^{2}$ and $T_{l c}$. All five statistics can be used to test hypothesis (1), and their asymptotic distributions are all standard normal distributions. We generate normal $x_{j}^{(l)}$ based on Model 1 and repeat the simulation 1,000 times to obtain 1,000 random points under the null hypothesis for all five statistics. The J-B test statistic represents a goodness-of-fit test to determine whether the skewness and kurtosis of sample data fit a standard normal distribution, and it is used here to determine whether the 1,000 random points follow a standard normal distribution. The J-B test statistic is expressed as follows:

$$
J B=\frac{n+m-1}{6}\left(S^{2}+\frac{1}{4}(C-3)^{2}\right),
$$

where $n$ is the number of observations, $S$ is the sample skewness, $C$ is the sample kurtosis, and $m$ is the number of regressors. The K-S test can also be used to compare a sample with a reference probability distribution and is defined as

$$
D_{n}=\sup _{x}\left|F_{n}(x)-F(x)\right|,
$$

where $F_{n}(x)$ is the empirical distribution function and $F(x)$ is a given cumulative distribution. When the value of $D_{n}$ is small, the sample is likely to obey the given distribution $F(x)$. Table 9 provides the 


\begin{tabular}{|c|c|c|c|c|c|c|c|c|c|c|}
\hline \multirow[t]{2}{*}{$\left(n_{1}, n_{2}\right)$} & \multirow{2}{*}{$\frac{\text { Method }}{\mathrm{p}}$} & \multicolumn{3}{|c|}{ Size $(\delta=0)$} & \multicolumn{3}{|c|}{ Power $(\delta=5)$} & \multicolumn{3}{|c|}{ Power $(\delta=10)$} \\
\hline & & 20 & 30 & 40 & 20 & 30 & 40 & 20 & 30 & 40 \\
\hline \multirow{3}{*}{$(25,35)$} & $T_{1}$ & 0.054 & 0.052 & 0.043 & 0.948 & 0.962 & 0.940 & 1 & 1 & 1 \\
\hline & $T_{2}$ & 0.045 & 0.040 & 0.045 & 0.325 & 0.442 & 0.620 & 0.869 & 0.960 & 0.997 \\
\hline & $T_{c l x}$ & 0.045 & 0.039 & 0.056 & 0.065 & 0.061 & 0.065 & 0.148 & 0.154 & 0.144 \\
\hline \multirow{4}{*}{$(50,70)$} & $\mathrm{p}$ & 40 & 60 & 80 & 40 & 60 & 80 & 40 & 60 & 80 \\
\hline & $T_{1}$ & 0.048 & 0.045 & 0.053 & 0.954 & 0.977 & 0.948 & 1 & 1 & 1 \\
\hline & $T_{2}$ & 0.055 & 0.048 & 0.053 & 0.248 & 0.455 & 0.582 & 0.828 & 0.962 & 0.992 \\
\hline & $T_{c l x}$ & 0.039 & 0.028 & 0.036 & 0.034 & 0.031 & 0.028 & 0.088 & 0.056 & 0.045 \\
\hline \multirow{4}{*}{$(100,140)$} & $\mathrm{p}$ & 80 & 120 & 160 & 80 & 120 & 160 & 80 & 120 & 160 \\
\hline & $T_{1}$ & 0.067 & 0.042 & 0.042 & 0.972 & 0.980 & 0.970 & 1 & 1 & 1 \\
\hline & $T_{2}$ & 0.051 & 0.039 & 0.052 & 0.252 & 0.472 & 0.606 & 0.780 & 0.959 & 0.996 \\
\hline & $T_{c l x}$ & 0.035 & 0.022 & 0.021 & 0.029 & 0.023 & 0.010 & 0.041 & 0.027 & 0.032 \\
\hline \multirow{4}{*}{$(200,280)$} & $\mathrm{p}$ & 160 & 240 & 320 & 160 & 240 & 320 & 160 & 240 & 320 \\
\hline & $T_{1}$ & 0.047 & 0.048 & 0.045 & 0.974 & 0.984 & 0.976 & 1 & 1 & 1 \\
\hline & $T_{2}$ & 0.054 & 0.050 & 0.048 & 0.270 & 0.441 & 0.587 & 0.736 & 0.948 & 0.986 \\
\hline & $T_{c l x}$ & 0.015 & 0.023 & 0.015 & 0.012 & 0.008 & 0.014 & 0.033 & 0.013 & 0.014 \\
\hline \multirow{4}{*}{$(400,560)$} & $\mathrm{p}$ & 320 & 480 & 640 & 320 & 480 & 640 & 320 & 480 & 640 \\
\hline & $T_{1}$ & 0.053 & 0.052 & 0.042 & 0.971 & 0.983 & 0.973 & 1 & 1 & 1 \\
\hline & $T_{2}$ & 0.051 & 0.053 & 0.040 & 0.231 & 0.439 & 0.559 & 0.740 & 0.952 & 0.993 \\
\hline & $T_{c l x}$ & 0.021 & 0.017 & 0.012 & 0.017 & 0.010 & 0.013 & 0.014 & 0.011 & 0.005 \\
\hline
\end{tabular}

Table 4: Empirical size and power from 1,000 repeated simulations comparing $T_{1}, T_{2}$ and $T_{c l x}$ based on Model 4 under the normal assumption.

p-values of the J-B and K-S tests for the five compared statistics. $\left(n_{1}, n_{2}, p\right)=(20,28,32),\left(n_{1}, n_{2}, p\right)=$ ${ }_{130}(20,32,28),\left(n_{1}, n_{2}, p\right)=(32,20,28)$, and $\left(n_{1}, n_{2}, p\right)=(32,28,20)$ represent the following cases: $y_{1}>$ $1, y_{2}>1 ; y_{1}>1, y_{2}<1 ; y<1, y_{2}>1$; and $y_{1}<1, y_{2}<1$, respectively. From Table 9 we find that the p-values exceed 0.05 , in most cases, which means we do not have sufficient evidence to reject the null hypothesis, especially when the dimension is high. Thus, we can conclude that the proposed statistics fit well for finite samples.

\section{Real data analysis}

In this section, we apply our method to an analysis of Standard and Poor's (S\&P) 500 index. The S\&P 500 index comprises 505 stocks trading on the American stock exchanges issued by 500 companies. There are ten Global Industry Classification Standard (GICS) sectors. After removing outlier data, each GICS sector includes many companies that reported 754 daily closing prices for three years.

On the stock market, volatility is the most frequently considered index, and it is critical for risk assessment. Volatility is the degree of variation in a trading price series over time as measured by the standard deviation of logarithmic returns. Many types of volatility exist, including historical volatility, which is a 

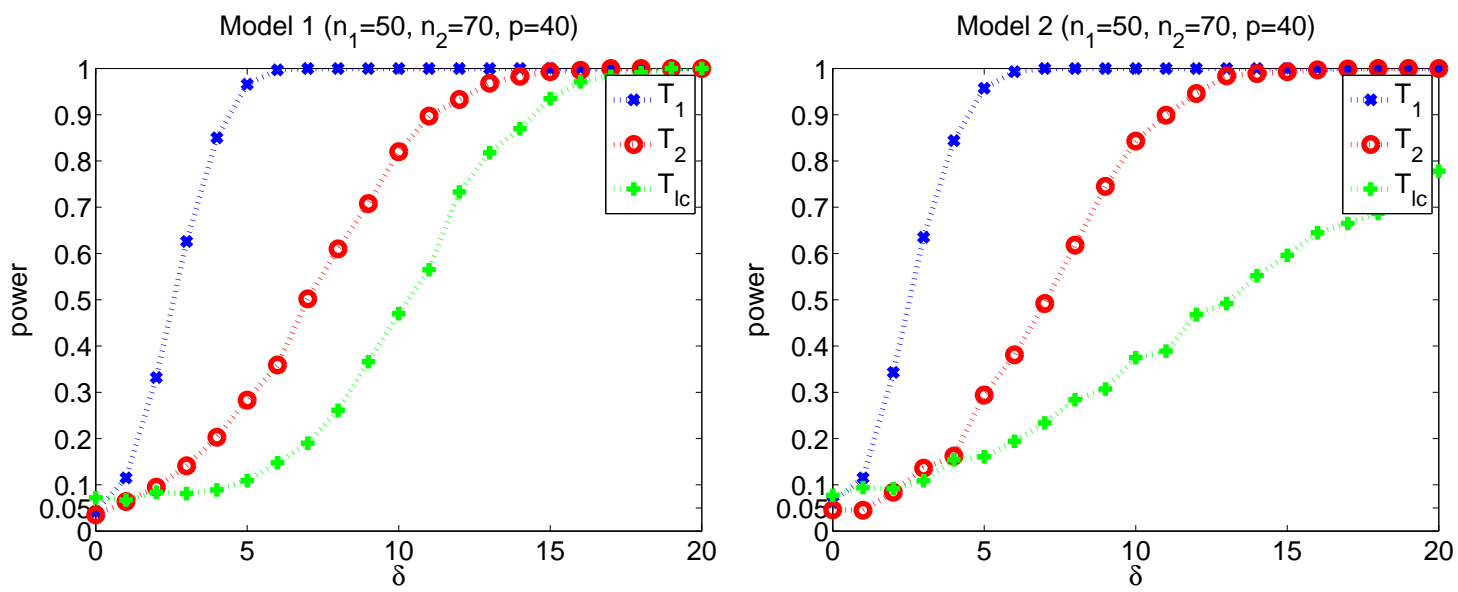

Figure 3: Scatter diagram of the empirical power for $T_{1}, T_{2}$ and $T_{l c}$ based on Model 1 and Model 2 under the normal assumption.
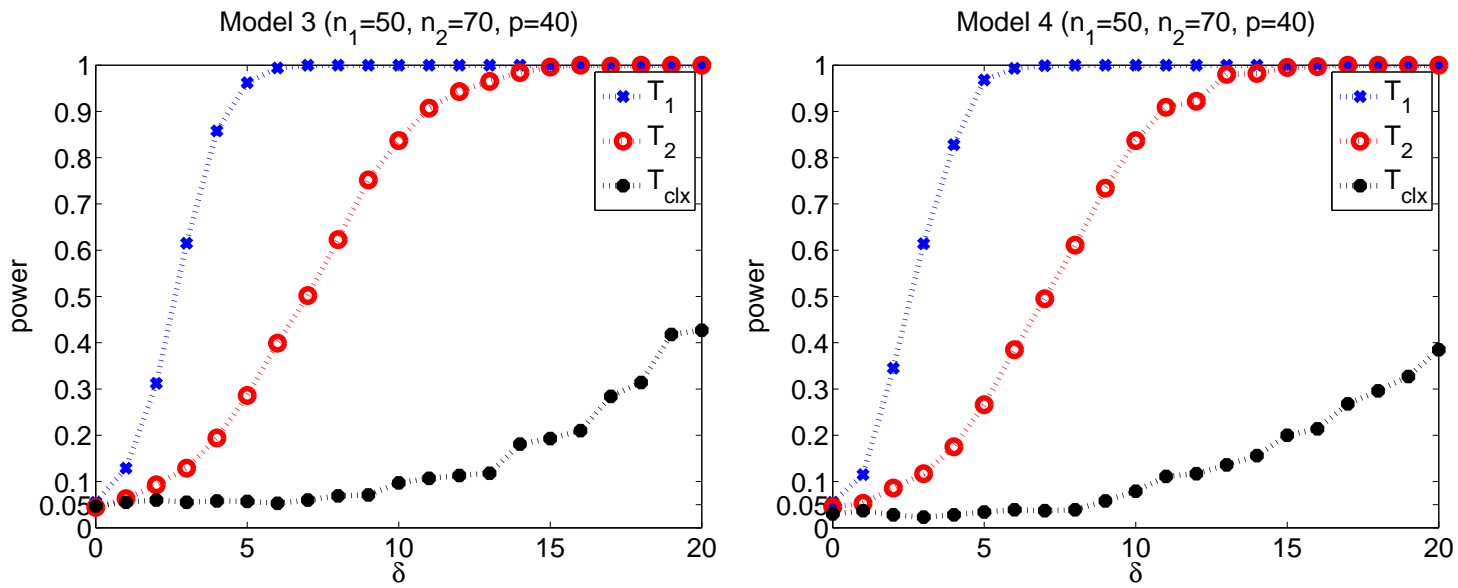

Figure 4: Scatter diagram of the empirical power for $T_{1}, T_{2}$ and $T_{c l x}$ based on Model 3 and Model 4 under the normal assumption.

time series consisting of past market prices, current volatility, and future volatility. In some sense, historical volatility provides a forecast of what a stock return will tend to be over a future period of time. We focus on the historical stock return volatility in 2 sectors: Energy and Information Technology. In each sector, our goal is to test whether the two covariance matrices of the stock returns are the same over time. The diagonal elements of the covariance matrix provide a rough representation of volatility, while the off-diagonal elements indicate the reciprocal effect between two stocks.

We consider the seasonal volatility of the daily returns of the selected stocks and choose the data from the first season of 2012 as sample 1 and the data from the second season in 2012 (S2Y12) to the last season in 2014 (S4Y14) as sample 2. We report the p-values of six tests based on the Energy sector in Table 10 and the Information Technology sector in Table 11. From these results, we find that most of the p-values are smaller than 0.05 . Thus, there is strong evidence that most of the covariance matrices are different, 

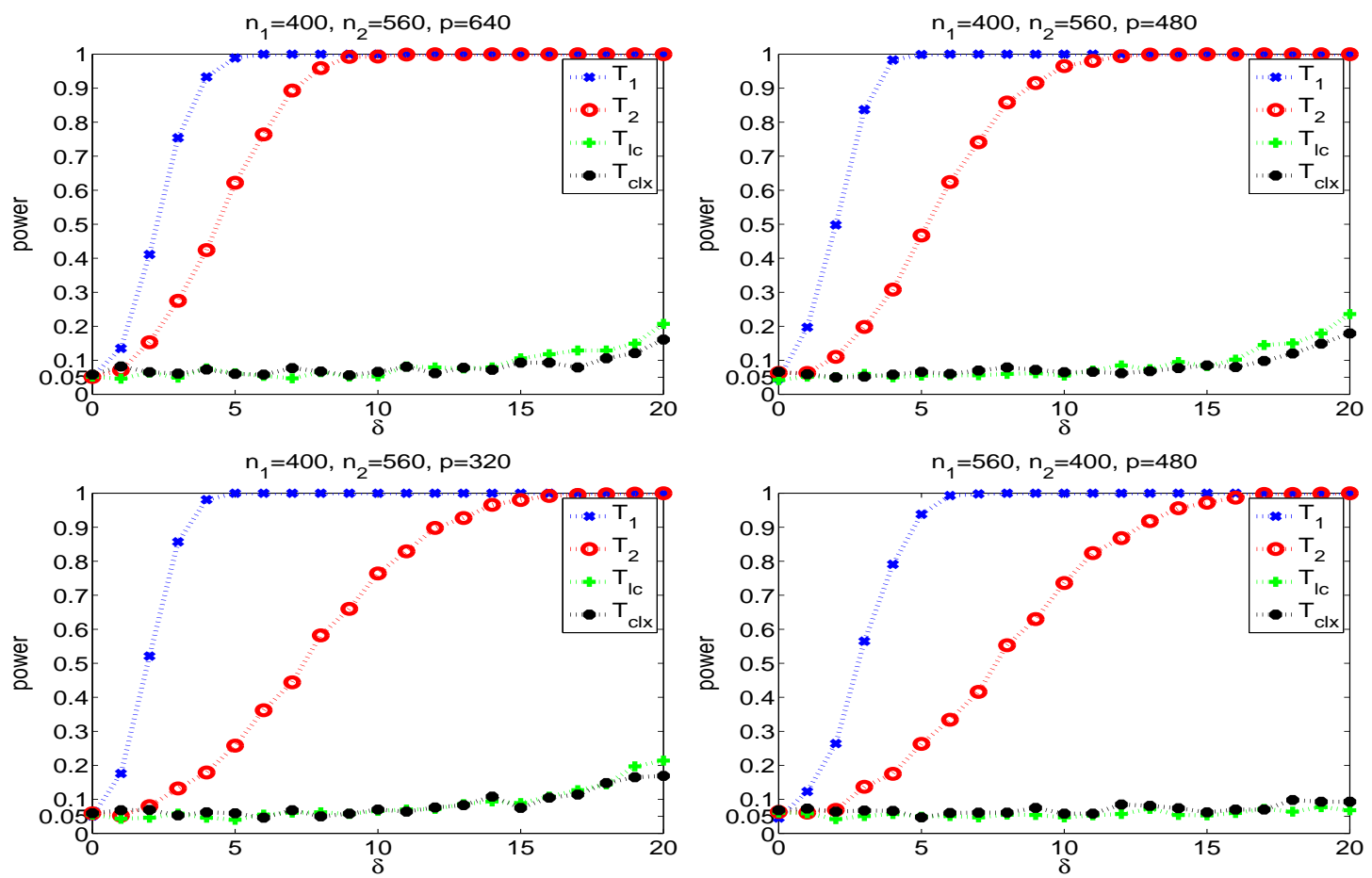

Figure 5: Scatter diagram of the empirical power for $T_{1}, T_{2}, T_{l c}$ and $T_{c l x}$ based on Model 1 under the uniform assumption.
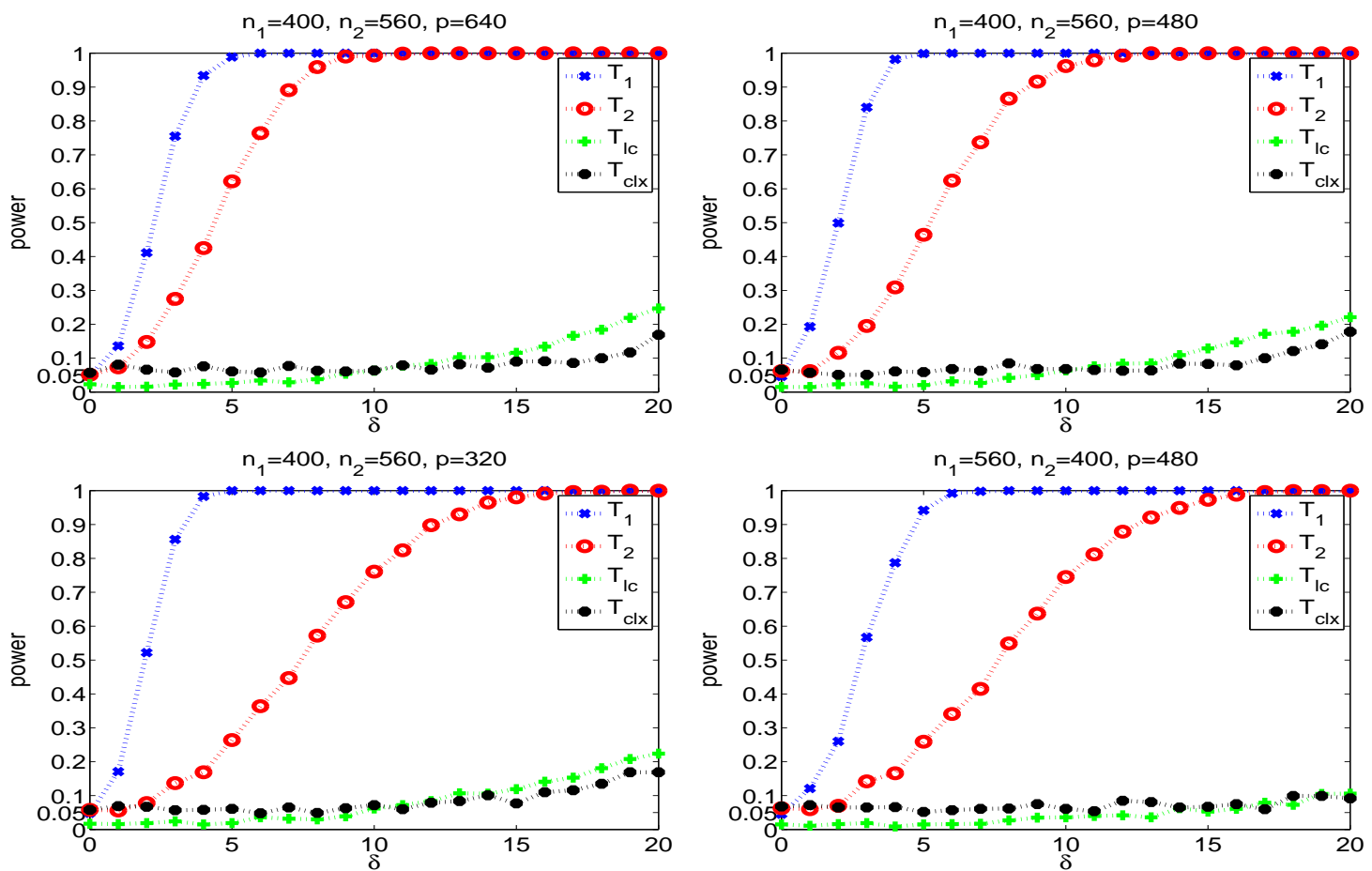

Figure 6: Scatter diagram of the empirical power for $T_{1}, T_{2}, T_{l c}$ and $T_{c l x}$ based on Model 2 under the uniform assumption. 

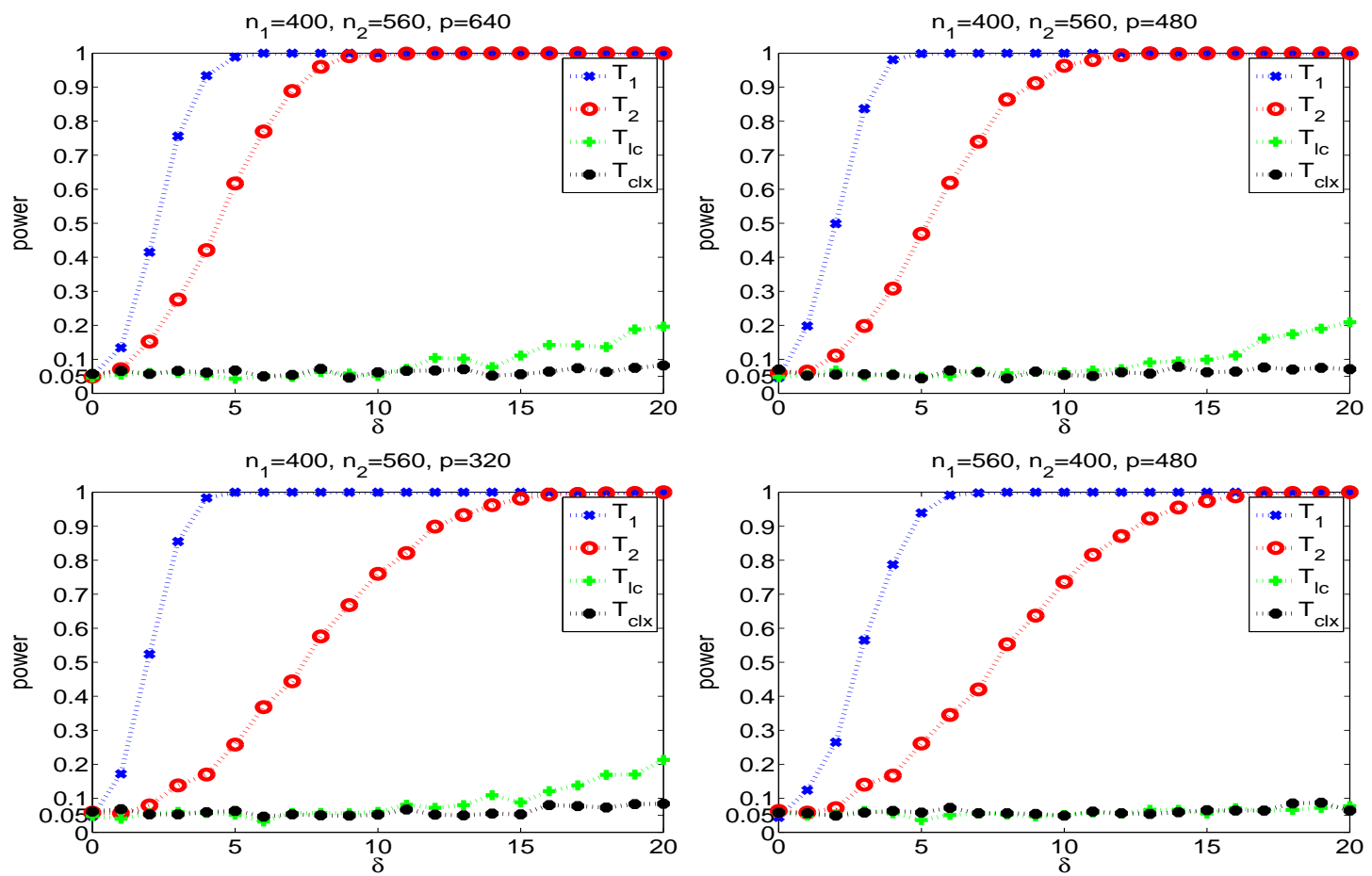

Figure 7: Scatter diagram of the empirical power for $T_{1}, T_{2}, T_{l c}$ and $T_{c l x}$ based on Model 3 under the uniform assumption.
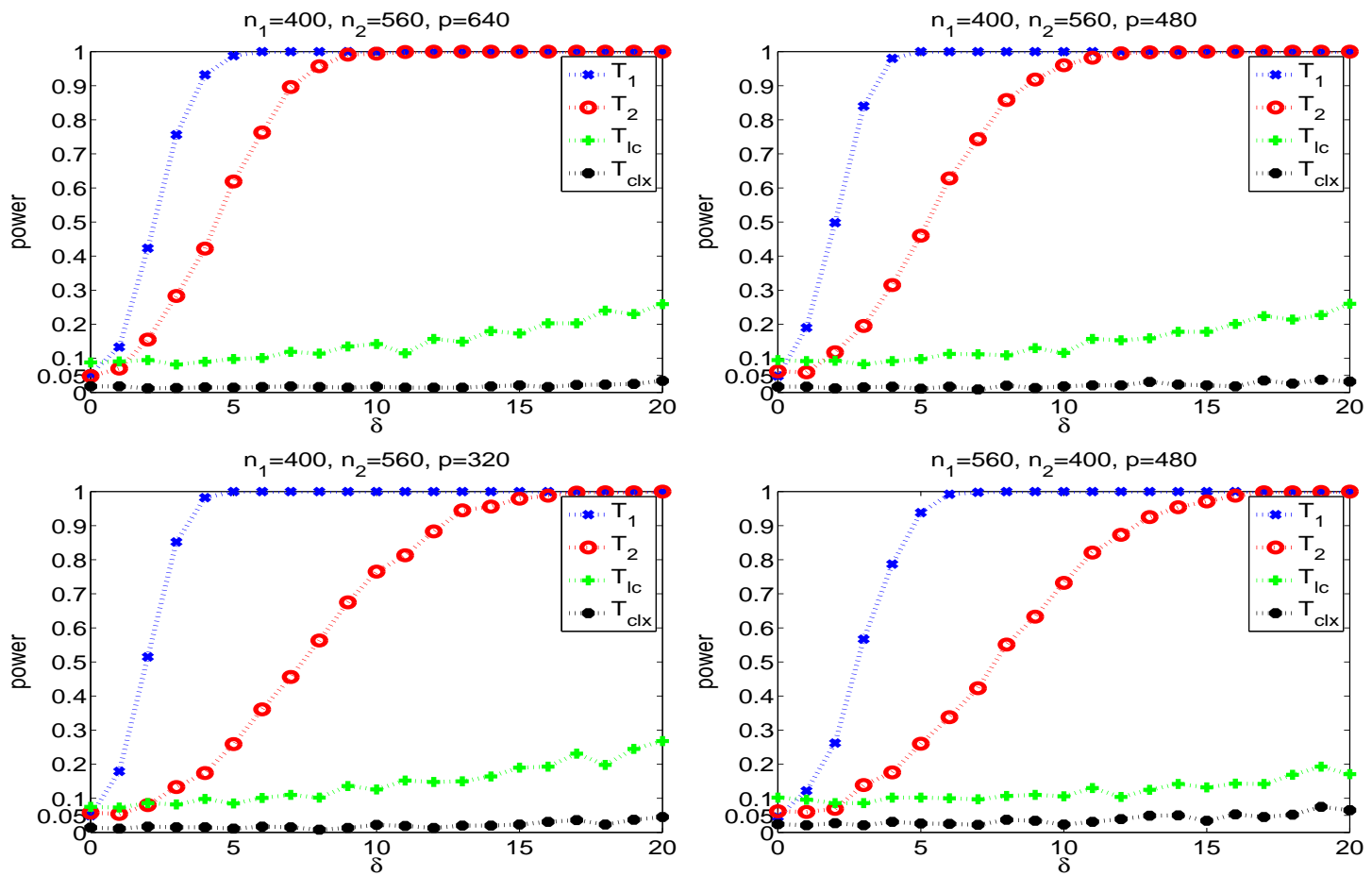

Figure 8: Scatter diagram of the empirical power for $T_{1}, T_{2}, T_{l c}$ and $T_{c l x}$ based on Model 4 under the uniform assumption. 

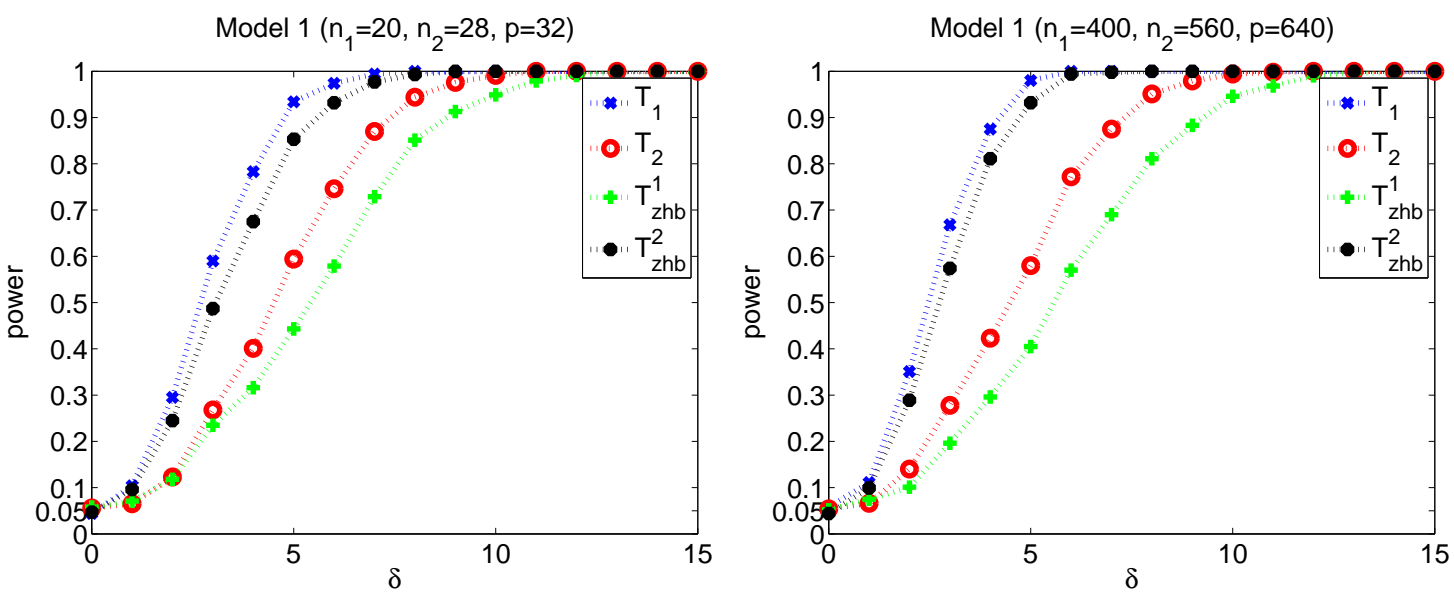

Figure 9: The empirical power for $T_{1}, T_{2}, T_{z h b}^{1}$ and $T_{z h b}^{2}$ under the normal assumption using Model 1.
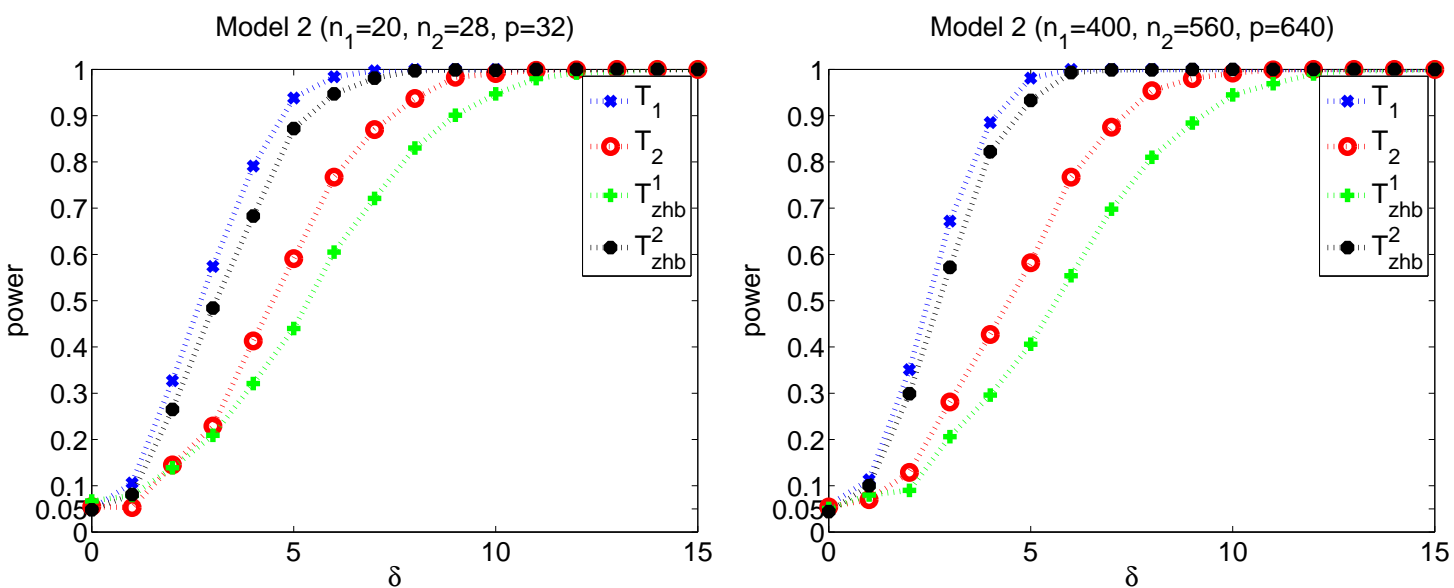

Figure 10: The empirical power for $T_{1}, T_{2}, T_{z h b}^{1}$ and $T_{z h b}^{2}$ under the normal assumption using Model 2.
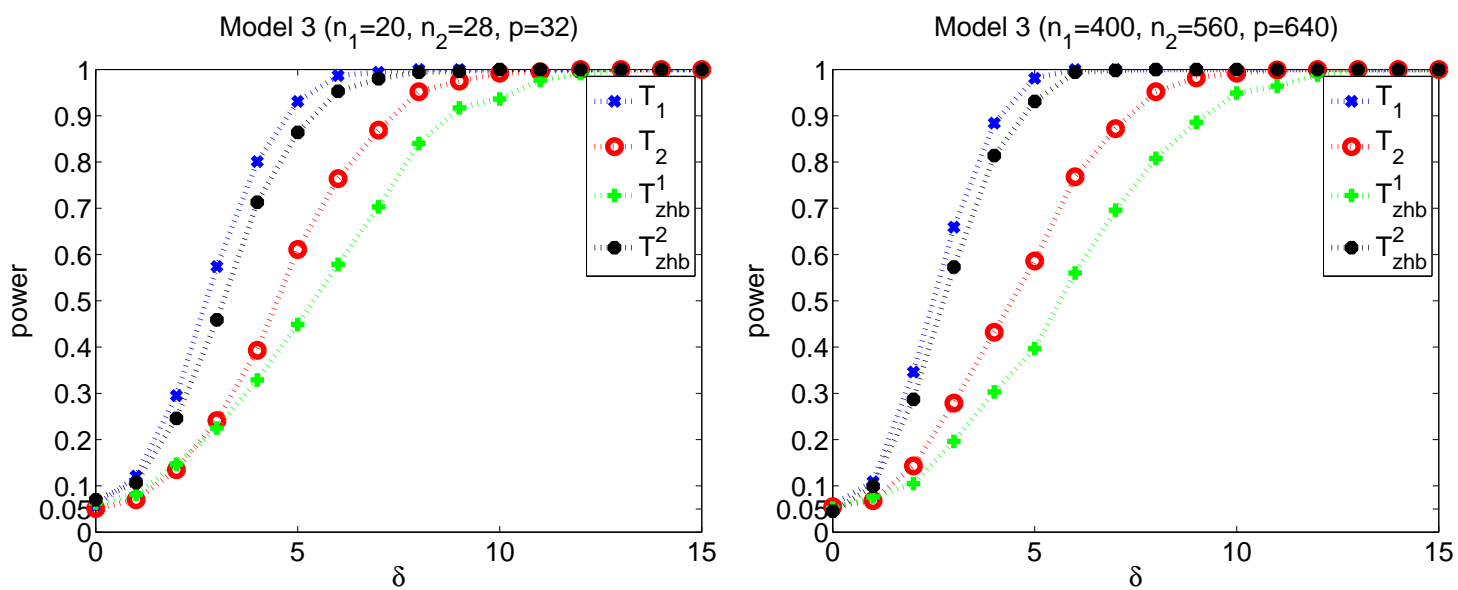

Figure 11: The empirical power for $T_{1}, T_{2}, T_{z h b}^{1}$ and $T_{z h b}^{2}$ under the normal assumption using Model 3. 


\begin{tabular}{|c|c|c|c|c|c|c|c|c|c|c|c|c|}
\hline \multirow{3}{*}{$\begin{array}{l}\left(n_{1}, n_{2}, p\right) \\
y_{1}>1, y_{2}> \\
1\end{array}$} & \multicolumn{3}{|c|}{$(50,70,80)$} & \multicolumn{3}{|c|}{$(100,140,160)$} & \multicolumn{3}{|c|}{$(200,280,320)$} & \multicolumn{3}{|c|}{$(400,560,640)$} \\
\hline & \multirow{2}{*}{$\begin{array}{l}\text { size } \\
\delta=0\end{array}$} & \multicolumn{2}{|c|}{ power } & \multirow{2}{*}{$\begin{array}{l}\text { size } \\
\delta=0 \\
\end{array}$} & \multicolumn{2}{|c|}{ power } & \multirow{2}{*}{$\begin{array}{l}\text { size } \\
\delta=0 \\
\end{array}$} & \multicolumn{2}{|c|}{ power } & \multirow{2}{*}{$\begin{array}{l}\text { size } \\
\delta=0 \\
\end{array}$} & \multicolumn{2}{|c|}{ power } \\
\hline & & $\delta=5$ & $\delta=10$ & & $\delta=5$ & $\delta=10$ & & $\delta=5$ & $\delta=10$ & & $\delta=5$ & $\delta=10$ \\
\hline$T_{1}$ & 0.043 & 0.991 & 1 & 0.054 & 0.987 & 1 & 0.053 & 0.993 & 1 & 0.052 & 0.990 & 1 \\
\hline$T_{2}$ & 0.055 & 0.622 & 1 & 0.049 & 0.623 & 0.997 & 0.058 & 0.620 & 0.995 & 0.058 & 0.643 & 0.996 \\
\hline$T_{l c}$ & 0.058 & 0.101 & 0.490 & 0.058 & 0.058 & 0.227 & 0.032 & 0.049 & 0.094 & 0.049 & 0.070 & 0.064 \\
\hline$T_{c l x}$ & 0.122 & 0.180 & 0.567 & 0.091 & 0.108 & 0.214 & 0.058 & 0.069 & 0.081 & 0.067 & 0.065 & 0.077 \\
\hline$\left(n_{1}, n_{2}, p\right)$ & \multicolumn{3}{|c|}{$(50,70,60)$} & \multicolumn{3}{|c|}{$(100,140,120)$} & \multicolumn{3}{|c|}{$(200,280,240)$} & \multicolumn{3}{|c|}{$(400,560,480)$} \\
\hline$y_{1}>1, y_{2}<$ & size & \multicolumn{2}{|c|}{ power } & size & \multicolumn{2}{|c|}{ power } & size & \multicolumn{2}{|c|}{ power } & size & \multicolumn{2}{|c|}{ power } \\
\hline 1 & $\delta=0$ & $\delta=5$ & $\delta=10$ & $\delta=0$ & $\delta=5$ & $\delta=10$ & $\delta=0$ & $\delta=5$ & $\delta=10$ & $\delta=0$ & $\delta=5$ & $\delta=10$ \\
\hline$T_{1}$ & 0.059 & 0.996 & 1 & 0.047 & 0.998 & 1 & 0.047 & 0.998 & 1 & 0.057 & 0.998 & 1 \\
\hline$T_{2}$ & 0.041 & 0.454 & 0.975 & 0.048 & 0.462 & 0.971 & 0.042 & 0.460 & 0.973 & 0.045 & 0.489 & 0.961 \\
\hline$T_{l c}$ & 0.055 & 0.099 & 0.518 & 0.048 & 0.062 & 0.200 & 0.054 & 0.072 & 0.113 & 0.049 & 0.055 & 0.062 \\
\hline$T_{c l x}$ & 0.120 & 0.161 & 0.587 & 0.076 & 0.121 & 0.230 & 0.078 & 0.083 & 0.122 & 0.060 & 0.048 & 0.072 \\
\hline$\left(n_{1}, n_{2}, p\right)$ & \multicolumn{3}{|c|}{$(70,50,60)$} & \multicolumn{3}{|c|}{$(140,100,120)$} & \multicolumn{3}{|c|}{$(280,200,240)$} & \multicolumn{3}{|c|}{$(560,400,480)$} \\
\hline$y_{1}<1, y_{2}>$ & size & \multicolumn{2}{|c|}{ power } & size & \multicolumn{2}{|c|}{ power } & size & \multicolumn{2}{|c|}{ power } & size & \multicolumn{2}{|c|}{ power } \\
\hline 1 & $\delta=0$ & $\delta=5$ & $\delta=10$ & $\delta=0$ & $\delta=5$ & $\delta=10$ & $\delta=0$ & $\delta=5$ & $\delta=10$ & $\delta=0$ & $\delta=5$ & $\delta=10$ \\
\hline$T_{1}$ & 0.052 & 0.928 & 1 & 0.057 & 0.945 & 1 & 0.048 & 0.946 & 1 & 0.053 & 0.931 & 1 \\
\hline$T_{2}$ & 0.048 & 0.224 & 0.572 & 0.048 & 0.251 & 0.684 & 0.032 & 0.241 & 0.715 & 0.060 & 0.241 & 0.748 \\
\hline$T_{l c}$ & 0.054 & 0.050 & 0.149 & 0.053 & 0.052 & 0.059 & 0.045 & 0.048 & 0.064 & 0.046 & 0.032 & 0.046 \\
\hline$T_{c l x}$ & 0.120 & 0.167 & 0.419 & 0.105 & 0.110 & 0.158 & 0.052 & 0.064 & 0.077 & 0.075 & 0.045 & 0.055 \\
\hline$\left(n_{1}, n_{2}, p\right)$ & & $50,70,40$ & & & 00,140, & & & $0,280,1$ & & & $0,560,3$ & \\
\hline$y_{1}<1, y_{2}<$ & size & & & size & & & size & & & size & & \\
\hline 1 & $\delta=0$ & $\delta=5$ & $\delta=10$ & $\delta=0$ & $\delta=5$ & $\delta=10$ & $\delta=0$ & $\delta=5$ & $\delta=10$ & $\delta=0$ & $\delta=5$ & $\delta=10$ \\
\hline$T_{1}$ & 0.047 & 0.994 & 1 & 0.050 & 0.999 & 1 & 0.047 & 1 & 1 & 0.045 & 0.999 & 1 \\
\hline$T_{2}$ & 0.055 & 0.279 & 0.860 & 0.044 & 0.269 & 0.810 & 0.052 & 0.281 & 0.779 & 0.046 & 0.243 & 0.755 \\
\hline$T_{l c}$ & 0.060 & 0.098 & 0.497 & 0.050 & 0.077 & 0.209 & 0.040 & 0.066 & 0.094 & 0.062 & 0.067 & 0.072 \\
\hline$T_{c l x}$ & 0.106 & 0.169 & 0.563 & 0.072 & 0.106 & 0.237 & 0.056 & 0.069 & 0.100 & 0.049 & 0.070 & 0.065 \\
\hline
\end{tabular}

Table 5: Empirical size and power for 1000 repeated simulations to compare $T_{1}, T_{2}, T_{l c}$ and $T_{c l x}$ based on Model 1 under the uniform assumption.

however, caution is advised when utilizing the assumption that the returns are identically distributed. In addition, there is no evidence to reject the null hypothesis for the first seasons of 2013 and 2014 in Table 10 . Thus, we suspect the presence of a periodic property in the covariance matrices. 


\begin{tabular}{|c|c|c|c|c|c|c|c|c|c|c|c|c|}
\hline \multirow{3}{*}{$\begin{array}{l}\left(n_{1}, n_{2}, p\right) \\
y_{1}>1, y_{2}> \\
1\end{array}$} & \multicolumn{3}{|c|}{$(50,70,80)$} & \multicolumn{3}{|c|}{$(100,140,160)$} & \multicolumn{3}{|c|}{$(200,280,320)$} & \multicolumn{3}{|c|}{$(400,560,640)$} \\
\hline & \multirow{2}{*}{$\begin{array}{l}\text { size } \\
\delta=0\end{array}$} & \multicolumn{2}{|c|}{ power } & \multirow{2}{*}{$\begin{array}{l}\text { size } \\
\delta=0\end{array}$} & \multicolumn{2}{|c|}{ power } & \multirow{2}{*}{$\begin{array}{l}\text { size } \\
\delta=0\end{array}$} & \multicolumn{2}{|c|}{ power } & \multirow{2}{*}{$\begin{array}{l}\text { size } \\
\delta=0\end{array}$} & \multicolumn{2}{|c|}{ power } \\
\hline & & $\delta=5$ & $\delta=10$ & & $\delta=5$ & $\delta=10$ & & $\delta=5$ & $\delta=10$ & & $\delta=5$ & $\delta=10$ \\
\hline$T_{1}$ & 0.048 & 0.992 & 1 & 0.061 & 0.989 & 1 & 0.060 & 0.996 & 1 & 0.046 & 0.991 & 1 \\
\hline$T_{2}$ & 0.048 & 0.623 & 0.999 & 0.053 & 0.645 & 0.997 & 0.044 & 0.591 & 1 & 0.052 & 0.604 & 0.992 \\
\hline$T_{l c}$ & 0.013 & 0.112 & 0.411 & 0.013 & 0.064 & 0.211 & 0.016 & 0.033 & 0.096 & 0.011 & 0.017 & 0.062 \\
\hline$T_{c l x}$ & 0.128 & 0.182 & 0.562 & 0.090 & 0.100 & 0.226 & 0.072 & 0.064 & 0.089 & 0.076 & 0.069 & 0.068 \\
\hline$\left(n_{1}, n_{2}, p\right)$ & \multicolumn{3}{|c|}{$(50,70,60)$} & \multicolumn{3}{|c|}{$(100,140,120)$} & \multicolumn{3}{|c|}{$(200,280,240)$} & \multicolumn{3}{|c|}{$(400,560,480)$} \\
\hline$y_{1}>1, y_{2}<$ & size & \multicolumn{2}{|c|}{ power } & size & \multicolumn{2}{|c|}{ power } & size & \multicolumn{2}{|c|}{ power } & size & \multicolumn{2}{|c|}{ power } \\
\hline 1 & $\delta=0$ & $\delta=5$ & $\delta=10$ & $\delta=0$ & $\delta=5$ & $\delta=10$ & $\delta=0$ & $\delta=5$ & $\delta=10$ & $\delta=0$ & $\delta=5$ & $\delta=10$ \\
\hline$T_{1}$ & 0.041 & 0.996 & 1 & 0.049 & 0.999 & 1 & 0.049 & 0.998 & 1 & 0.045 & 0.998 & 1 \\
\hline$T_{2}$ & 0.045 & 0.459 & 0.977 & 0.045 & 0.487 & 0.969 & 0.050 & 0.463 & 0.963 & 0.033 & 0.481 & 0.968 \\
\hline$T_{l c}$ & 0.014 & 0.115 & 0.409 & 0.006 & 0.060 & 0.237 & 0.015 & 0.024 & 0.140 & 0.018 & 0.031 & 0.062 \\
\hline$T_{c l x}$ & 0.108 & 0.152 & 0.578 & 0.077 & 0.102 & 0.228 & 0.077 & 0.066 & 0.107 & 0.056 & 0.059 & 0.060 \\
\hline$\left(n_{1}, n_{2}, p\right)$ & \multicolumn{3}{|c|}{$(70,50,60)$} & \multicolumn{3}{|c|}{$(140,100,120)$} & \multicolumn{3}{|c|}{$(280,200,240)$} & \multicolumn{3}{|c|}{$(560,400,480)$} \\
\hline$y_{1}<1, y_{2}>$ & size & \multicolumn{2}{|c|}{ power } & size & \multicolumn{2}{|c|}{ power } & size & \multicolumn{2}{|c|}{ power } & size & \multicolumn{2}{|c|}{ power } \\
\hline 1 & $\delta=0$ & $\delta=5$ & $\delta=10$ & $\delta=0$ & $\delta=5$ & $\delta=10$ & $\delta=0$ & $\delta=5$ & $\delta=10$ & $\delta=0$ & $\delta=5$ & $\delta=10$ \\
\hline$T_{1}$ & 0.047 & 0.941 & 1 & 0.042 & 0.941 & 1 & 0.051 & 0.936 & 1 & 0.055 & 0.931 & 1 \\
\hline$T_{2}$ & 0.048 & 0.220 & 0.560 & 0.046 & 0.241 & 0.666 & 0.043 & 0.269 & 0.711 & 0.057 & 0.258 & 0.756 \\
\hline$T_{l c}$ & 0.015 & 0.055 & 0.186 & 0.013 & 0.028 & 0.106 & 0.019 & 0.021 & 0.066 & 0.015 & 0.022 & 0.041 \\
\hline$T_{c l x}$ & 0.122 & 0.168 & 0.392 & 0.103 & 0.099 & 0.158 & 0.073 & 0.059 & 0.076 & 0.066 & 0.060 & 0.053 \\
\hline$\left(n_{1}, n_{2}, p\right)$ & & $50,70,4$ & & & $00,140,8$ & & & $0,280,1$ & & & $0,560,3$ & \\
\hline$y_{1}<1, y_{2}<$ & size & & & size & & & size & & & size & & \\
\hline 1 & $\delta=0$ & $\delta=5$ & $\delta=10$ & $\delta=0$ & $\delta=5$ & $\delta=10$ & $\delta=0$ & $\delta=5$ & $\delta=10$ & $\delta=0$ & $\delta=5$ & $\delta=10$ \\
\hline$T_{1}$ & 0.048 & 0.996 & 1 & 0.058 & 0.998 & 1 & 0.039 & 0.999 & 1 & 0.042 & 1 & 1 \\
\hline$T_{2}$ & 0.045 & 0.283 & 0.864 & 0.050 & 0.264 & 0.812 & 0.046 & 0.282 & 0.767 & 0.042 & 0.252 & 0.788 \\
\hline$T_{l c}$ & 0.012 & 0.115 & 0.388 & 0.018 & 0.067 & 0.220 & 0.008 & 0.037 & 0.112 & 0.008 & 0.019 & 0.066 \\
\hline$T_{c l x}$ & 0.122 & 0.171 & 0.589 & 0.077 & 0.096 & 0.255 & 0.072 & 0.055 & 0.107 & 0.059 & 0.066 & 0.070 \\
\hline
\end{tabular}

Table 6: Empirical size and power for 1000 repeated simulations to compare $T_{1}, T_{2}, T_{l c}$ and $T_{c l x}$ based on Model 2 under the uniform assumption.
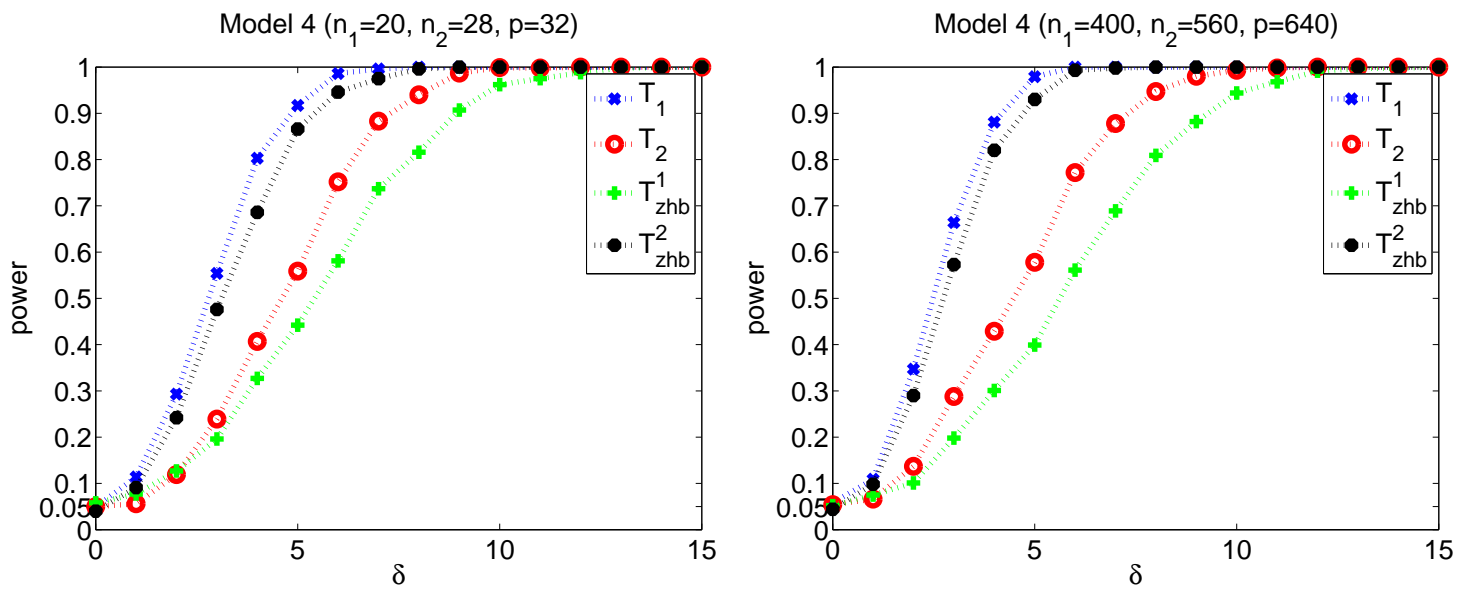

Figure 12: The empirical power for $T_{1}, T_{2}, T_{z h b}^{1}$ and $T_{z h b}^{2}$ under the normal assumption using Model 4. 


\begin{tabular}{|c|c|c|c|c|c|c|c|c|c|c|c|c|}
\hline \multirow{3}{*}{$\begin{array}{l}\left(n_{1}, n_{2}, p\right) \\
y_{1}>1, y_{2}> \\
1\end{array}$} & \multicolumn{3}{|c|}{$(50,70,80)$} & \multicolumn{3}{|c|}{$(100,140,160)$} & \multicolumn{3}{|c|}{$(200,280,320)$} & \multicolumn{3}{|c|}{$(400,560,640)$} \\
\hline & \multirow{2}{*}{$\begin{array}{l}\text { size } \\
\delta=0\end{array}$} & \multicolumn{2}{|c|}{ power } & \multirow{2}{*}{$\begin{array}{l}\text { size } \\
\delta=0\end{array}$} & \multicolumn{2}{|c|}{ power } & \multirow{2}{*}{$\begin{array}{c}\text { size } \\
\delta=0\end{array}$} & \multicolumn{2}{|c|}{ power } & \multirow{2}{*}{$\begin{array}{c}\text { size } \\
\delta=0\end{array}$} & \multicolumn{2}{|c|}{ power } \\
\hline & & $\delta=5$ & $\delta=10$ & & $\delta=5$ & $\delta=10$ & & $\delta=5$ & $\delta=10$ & & $\delta=5$ & $\delta=10$ \\
\hline$T_{1}$ & 0.040 & 0.991 & 1 & 0.053 & 0.990 & 1 & 0.046 & 0.986 & 1 & 0.060 & 0.992 & 1 \\
\hline$T_{2}$ & 0.053 & 0.621 & 1 & 0.056 & 0.643 & 0.996 & 0.054 & 0.620 & 0.996 & 0.055 & 0.602 & 0.996 \\
\hline$T_{l c}$ & 0.053 & 0.113 & 0.475 & 0.060 & 0.077 & 0.245 & 0.056 & 0.058 & 0.098 & 0.042 & 0.059 & 0.064 \\
\hline$T_{c l x}$ & 0.096 & 0.095 & 0.222 & 0.070 & 0.088 & 0.108 & 0.063 & 0.062 & 0.071 & 0.044 & 0.048 & 0.066 \\
\hline$\left(n_{1}, n_{2}, p\right)$ & \multicolumn{3}{|c|}{$(50,70,60)$} & \multicolumn{3}{|c|}{$(100,140,120)$} & \multicolumn{3}{|c|}{$(200,280,240)$} & \multicolumn{3}{|c|}{$(400,560,480)$} \\
\hline$y_{1}>1, y_{2}<$ & size & \multicolumn{2}{|c|}{ power } & size & \multicolumn{2}{|c|}{ power } & size & \multicolumn{2}{|c|}{ power } & size & \multicolumn{2}{|c|}{ power } \\
\hline 1 & $\delta=0$ & $\delta=5$ & $\delta=10$ & $\delta=0$ & $\delta=5$ & $\delta=10$ & $\delta=0$ & $\delta=5$ & $\delta=10$ & $\delta=0$ & $\delta=5$ & $\delta=10$ \\
\hline$T_{1}$ & 0.063 & 0.996 & 1 & 0.063 & 0.999 & 1 & 0.042 & 0.999 & 1 & 0.043 & 0.998 & 1 \\
\hline$T_{2}$ & 0.056 & 0.469 & 0.982 & 0.045 & 0.481 & 0.974 & 0.051 & 0.478 & 0.978 & 0.051 & 0.460 & 0.962 \\
\hline$T_{l c}$ & 0.063 & 0.124 & 0.483 & 0.068 & 0.077 & 0.192 & 0.043 & 0.054 & 0.104 & 0.058 & 0.054 & 0.063 \\
\hline$T_{c l x}$ & 0.082 & 0.109 & 0.240 & 0.088 & 0.076 & 0.098 & 0.060 & 0.049 & 0.063 & 0.064 & 0.047 & 0.064 \\
\hline$\left(n_{1}, n_{2}, p\right)$ & \multicolumn{3}{|c|}{$(70,50,60)$} & \multicolumn{3}{|c|}{$(140,100,120)$} & \multicolumn{3}{|c|}{$(280,200,240)$} & \multicolumn{3}{|c|}{$(560,400,480)$} \\
\hline$y_{1}<1, y_{2}>$ & size & \multicolumn{2}{|c|}{ power } & size & \multicolumn{2}{|c|}{ power } & size & \multicolumn{2}{|c|}{ power } & size & \multicolumn{2}{|c|}{ power } \\
\hline 1 & $\delta=0$ & $\delta=5$ & $\delta=10$ & $\delta=0$ & $\delta=5$ & $\delta=10$ & $\delta=0$ & $\delta=5$ & $\delta=10$ & $\delta=0$ & $\delta=5$ & $\delta=10$ \\
\hline$T_{1}$ & 0.043 & 0.937 & 1 & 0.038 & 0.930 & 1 & 0.044 & 0.946 & 1 & 0.052 & 0.940 & 1 \\
\hline$T_{2}$ & 0.036 & 0.228 & 0.596 & 0.038 & 0.218 & 0.664 & 0.040 & 0.238 & 0.716 & 0.039 & 0.277 & 0.720 \\
\hline$T_{l c}$ & 0.058 & 0.065 & 0.172 & 0.058 & 0.058 & 0.095 & 0.051 & 0.053 & 0.068 & 0.051 & 0.064 & 0.051 \\
\hline$T_{c l x}$ & 0.091 & 0.113 & 0.241 & 0.069 & 0.073 & 0.107 & 0.050 & 0.055 & 0.061 & 0.051 & 0.065 & 0.051 \\
\hline$\left(n_{1}, n_{2}, p\right)$ & & $50,70,40$ & & & 00,140, & & & $0,280,1$ & & & $0,560,3$ & \\
\hline$y_{1}<1, y_{2}<$ & size & & & size & & & size & & & size & & \\
\hline 1 & $\delta=0$ & $\delta=5$ & $\delta=10$ & $\delta=0$ & $\delta=5$ & $\delta=10$ & $\delta=0$ & $\delta=5$ & $\delta=10$ & $\delta=0$ & $\delta=5$ & $\delta=10$ \\
\hline$T_{1}$ & 0.065 & 0.995 & 1 & 0.045 & 0.997 & 1 & 0.049 & 1 & 1 & 0.042 & 1 & 1 \\
\hline$T_{2}$ & 0.042 & 0.293 & 0.854 & 0.054 & 0.287 & 0.774 & 0.051 & 0.281 & 0.777 & 0.042 & 0.251 & 0.757 \\
\hline$T_{l c}$ & 0.054 & 0.120 & 0.445 & 0.059 & 0.087 & 0.193 & 0.055 & 0.049 & 0.105 & 0.040 & 0.059 & 0.059 \\
\hline$T_{c l x}$ & 0.103 & 0.094 & 0.235 & 0.063 & 0.072 & 0.093 & 0.066 & 0.058 & 0.075 & 0.061 & 0.053 & 0.054 \\
\hline
\end{tabular}

Table 7: Empirical size and power for 1000 repeated simulations to compare $T_{1}, T_{2}, T_{l c}$ and $T_{c l x}$ based on Model 3 under the uniform assumption. 


\begin{tabular}{|c|c|c|c|c|c|c|c|c|c|c|c|c|}
\hline \multirow{3}{*}{$\begin{array}{l}\left(n_{1}, n_{2}, p\right) \\
y_{1}>1, y_{2}> \\
1\end{array}$} & \multicolumn{3}{|c|}{$(50,70,80)$} & \multicolumn{3}{|c|}{$(100,140,160)$} & \multicolumn{3}{|c|}{$(200,280,320)$} & \multicolumn{3}{|c|}{$(400,560,640)$} \\
\hline & \multirow{2}{*}{$\begin{array}{l}\text { size } \\
\delta=0\end{array}$} & \multicolumn{2}{|c|}{ power } & \multirow{2}{*}{$\begin{array}{l}\text { size } \\
\delta=0\end{array}$} & \multicolumn{2}{|c|}{ power } & \multirow{2}{*}{$\begin{array}{l}\text { size } \\
\delta=0\end{array}$} & \multicolumn{2}{|c|}{ power } & \multirow{2}{*}{$\begin{array}{c}\text { size } \\
\delta=0\end{array}$} & \multicolumn{2}{|c|}{ power } \\
\hline & & $\delta=5$ & $\delta=10$ & & $\delta=5$ & $\delta=10$ & & $\delta=5$ & $\delta=10$ & & $\delta=5$ & $\delta=10$ \\
\hline$T_{1}$ & 0.060 & 0.992 & 1 & 0.055 & 0.988 & 1 & 0.066 & 0.995 & 1 & 0.050 & 0.994 & 1 \\
\hline$T_{2}$ & 0.057 & 0.616 & 0.998 & 0.049 & 0.606 & 0.996 & 0.053 & 0.652 & 0.993 & 0.051 & 0.603 & 0.997 \\
\hline$T_{l c}$ & 0.082 & 0.156 & 0.367 & 0.096 & 0.122 & 0.251 & 0.087 & 0.122 & 0.148 & 0.073 & 0.098 & 0.516 \\
\hline$T_{c l x}$ & 0.041 & 0.030 & 0.065 & 0.035 & 0.023 & 0.036 & 0.022 & 0.013 & 0.021 & 0.065 & 0.011 & 0.216 \\
\hline$\left(n_{1}, n_{2}, p\right)$ & \multicolumn{3}{|c|}{$(50,70,60)$} & \multicolumn{3}{|c|}{$(100,140,120)$} & \multicolumn{3}{|c|}{$(200,280,240)$} & \multicolumn{3}{|c|}{$(400,560,480)$} \\
\hline$y_{1}>1, y_{2}<$ & size & \multicolumn{2}{|c|}{ power } & size & \multicolumn{2}{|c|}{ power } & size & \multicolumn{2}{|c|}{ power } & size & \multicolumn{2}{|c|}{ power } \\
\hline 1 & $\delta=0$ & $\delta=5$ & $\delta=10$ & $\delta=0$ & $\delta=5$ & $\delta=10$ & $\delta=0$ & $\delta=5$ & $\delta=10$ & $\delta=0$ & $\delta=5$ & $\delta=10$ \\
\hline$T_{1}$ & 0.051 & 0.996 & 1 & 0.057 & 1 & 1 & 0.050 & 0.999 & 1 & 0.052 & 0.999 & 1 \\
\hline$T_{2}$ & 0.053 & 0.464 & 0.979 & 0.047 & 0.444 & 0.978 & 0.053 & 0.473 & 0.968 & 0.053 & 0.438 & 0.974 \\
\hline$T_{l c}$ & 0.082 & 0.175 & 0.363 & 0.076 & 0.121 & 0.259 & 0.083 & 0.104 & 0.181 & 0.094 & 0.121 & 0.508 \\
\hline$T_{c l x}$ & 0.037 & 0.035 & 0.083 & 0.036 & 0.024 & 0.044 & 0.027 & 0.012 & 0.026 & 0.058 & 0.014 & 0.251 \\
\hline$\left(n_{1}, n_{2}, p\right)$ & \multicolumn{3}{|c|}{$(70,50,60)$} & \multicolumn{3}{|c|}{$(140,100,120)$} & \multicolumn{3}{|c|}{$(280,200,240)$} & \multicolumn{3}{|c|}{$(560,400,480)$} \\
\hline$y_{1}<1, y_{2}>$ & size & \multicolumn{2}{|c|}{ power } & size & \multicolumn{2}{|c|}{ power } & size & \multicolumn{2}{|c|}{ power } & size & \multicolumn{2}{|c|}{ power } \\
\hline 1 & $\delta=0$ & $\delta=5$ & $\delta=10$ & $\delta=0$ & $\delta=5$ & $\delta=10$ & $\delta=0$ & $\delta=5$ & $\delta=10$ & $\delta=0$ & $\delta=5$ & $\delta=10$ \\
\hline$T_{1}$ & 0.046 & 0.935 & 1 & 0.047 & 0.939 & 1 & 0.048 & 0.941 & 1 & 0.054 & 0.946 & 1 \\
\hline$T_{2}$ & 0.052 & 0.224 & 0.605 & 0.053 & 0.248 & 0.671 & 0.044 & 0.223 & 0.726 & 0.054 & 0.268 & 0.906 \\
\hline$T_{l c}$ & 0.075 & 0.113 & 0.222 & 0.081 & 0.108 & 0.184 & 0.074 & 0.099 & 0.132 & 0.077 & 0.094 & 0.310 \\
\hline$T_{c l x}$ & 0.029 & 0.075 & 0.138 & 0.030 & 0.050 & 0.084 & 0.012 & 0.035 & 0.055 & 0.081 & 0.019 & 0.282 \\
\hline$\left(n_{1}, n_{2}, p\right)$ & & $50,70,40$ & & & 00,140, & & & $0,280,1$ & & & $0,560,3$ & \\
\hline$y_{1}<1, y_{2}<$ & size & & & size & & & size & & & size & & \\
\hline 1 & $\delta=0$ & $\delta=5$ & $\delta=10$ & $\delta=0$ & $\delta=5$ & $\delta=10$ & $\delta=0$ & $\delta=5$ & $\delta=10$ & $\delta=0$ & $\delta=5$ & $\delta=10$ \\
\hline$T_{1}$ & 0.062 & 0.995 & 1 & 0.045 & 0.998 & 1 & 0.052 & 1 & 1 & 0.053 & 0.999 & 0.999 \\
\hline$T_{2}$ & 0.063 & 0.287 & 0.861 & 0.066 & 0.274 & 0.802 & 0.046 & 0.258 & 0.789 & 0.047 & 0.261 & 0.452 \\
\hline$T_{l c}$ & 0.088 & 0.153 & 0.381 & 0.076 & 0.129 & 0.229 & 0.087 & 0.096 & 0.157 & 0.088 & 0.102 & 0.536 \\
\hline$T_{c l x}$ & 0.048 & 0.049 & 0.109 & 0.026 & 0.026 & 0.041 & 0.024 & 0.020 & 0.024 & 0.063 & 0.015 & 0.273 \\
\hline
\end{tabular}

Table 8: Empirical size and power for 1000 repeated simulations to compare $T_{1}, T_{2}, T_{l c}$ and $T_{c l x}$ based on Model 4 under the uniform assumption. 


\begin{tabular}{|c|c|c|c|c|c|c|c|c|}
\hline \multirow{3}{*}{ Method } & \multicolumn{6}{|c|}{ Low-dimensional $\left(n_{1}, n_{2}, p\right)$} & & \\
\hline & \multicolumn{2}{|c|}{$(20,28,32)$} & \multicolumn{2}{|c|}{$(20,32,28)$} & \multicolumn{2}{|c|}{$(32,20,28)$} & \multicolumn{2}{|c|}{$(32,28,20)$} \\
\hline & J-B test & K-S test & J-B test & $\mathrm{K}-\mathrm{S}$ test & J-B test & $\mathrm{K}-\mathrm{S}$ test & J-B test & $\mathrm{K}-\mathrm{S}$ test \\
\hline$T_{1}$ & 0.986 & 0.291 & 0.570 & 0.243 & 0.711 & 0.582 & 0.308 & 0.438 \\
\hline$T_{z h b}^{1}$ & 0 & 0.473 & 0.010 & 0.004 & 0.016 & 0.030 & 0.303 & 0.131 \\
\hline$T_{2}$ & 0.334 & 0.748 & 0.573 & 0.198 & 0.217 & 0.102 & 0.936 & 0.053 \\
\hline$T_{z h b}^{2}$ & 0.059 & 0.002 & 0.492 & 0.243 & 0.115 & 0.021 & 0.075 & 0.043 \\
\hline$T_{l c}$ & 0.097 & 0.049 & 0.534 & 0.019 & 0.630 & 0.103 & 0.942 & 0.141 \\
\hline \multirow{3}{*}{ Method } & \multicolumn{6}{|c|}{ High-dimensional $\left(n_{1}, n_{2}, p\right)$} & & \\
\hline & \multicolumn{2}{|c|}{$(400,560,640)$} & \multicolumn{2}{|c|}{$(400,640,560)$} & \multicolumn{2}{|c|}{$(640,400,560)$} & \multicolumn{2}{|c|}{$(640,560,400)$} \\
\hline & J-B test & K-S test & J-B test & K-S test & $\mathrm{J}$-B test & K-S test & $\mathrm{J}$-B test & $\mathrm{K}$-S test \\
\hline$T_{1}$ & 0.406 & 0.167 & 0.933 & 0.395 & 0.952 & 0.686 & 0.958 & 0.613 \\
\hline$T_{z h b}^{1}$ & 0.197 & 0.556 & 0.628 & 0.505 & 0.084 & 0.022 & 0.468 & 0.951 \\
\hline$T_{2}$ & 0.094 & 0.326 & 0.366 & 0.534 & 0.568 & 0.159 & 0.581 & 0.978 \\
\hline$T_{z h b}^{2}$ & 0.276 & 0.328 & 0.996 & 0.647 & 0.432 & 0.464 & 0.616 & 0.432 \\
\hline$T_{l c}$ & 0.892 & 0.181 & 0.369 & 0.252 & 0.400 & 0.863 & 0.192 & 0.772 \\
\hline
\end{tabular}

Table 9: P-values of the J-B and K-S tests for $T_{1}, T_{2}, T_{z h b}^{1}, T_{z h b}^{2}$ and $T_{l c}$ under the normal assumption.

\begin{tabular}{cccccccccccc}
\hline Season & S2Y12 & S3Y12 & S4Y12 & S1Y13 & S2Y13 & S3Y13 & S4Y13 & S1Y14 & S2Y14 & S3Y14 & S4Y14 \\
\hline$T_{1}$ & 0 & 0 & 0.005 & 0.237 & 0.116 & 0.173 & 0.290 & 0.491 & 0.177 & 0.033 & 0.165 \\
\hline$T_{2}$ & 0 & 0 & 0 & 0.256 & 0.378 & 0.153 & 0 & 0.030 & 0.317 & 0.016 & 0.002 \\
\hline$T_{z h b}^{1}$ & 0 & 0 & 0.001 & 0.095 & 0.078 & 0.036 & 0 & 0.001 & 0.029 & 0 & 0 \\
\hline$T_{z h b}^{2}$ & 0 & 0.001 & 0.007 & 0.340 & 0.051 & 0.119 & 0.495 & 0.400 & 0.194 & 0.044 & 0.057 \\
\hline$T_{l c}$ & 0 & 0 & 0.162 & 0.147 & 0 & 0 & 0 & 0.007 & 0 & 0 & 0 \\
\hline$T_{c l x}$ & 0.004 & 0.035 & 0.129 & 0.404 & 0.002 & 0.065 & 0.003 & 0.110 & 0.027 & 0.145 & 0.003 \\
\hline
\end{tabular}

Table 10: P-values from the tests of the two covariance matrices of daily stock returns in the Energy sector. 


\begin{tabular}{cccccccccccc}
\hline Season & S2Y12 & S3Y12 & S4Y12 & S1Y13 & S2Y13 & S3Y13 & S4Y13 & S1Y14 & S2Y14 & S3Y14 & S4Y14 \\
\hline$T_{1}$ & 0.1672 & 0.0110 & 0.0024 & 0.0025 & 0 & 0.0011 & 0.3997 & 0 & 0 & 0.0015 & 0.4679 \\
\hline$T_{2}$ & 0 & 0 & 0 & 0 & 0 & 0 & 0 & 0 & 0 & 0 & 0 \\
\hline$T_{z h b}^{1}$ & 0 & 0 & 0 & 0 & 0 & 0 & 0 & 0 & 0 & 0 & 0 \\
\hline$T_{z h b}^{2}$ & 0.0102 & 0.0001 & 0.2511 & 0. & 0 & 0 & 0.0503 & 0 & 0 & 0 & 0.0789 \\
\hline$T_{l c}$ & 0 & 0.2761 & 0.0203 & 0 & 0.1036 & 0.0066 & 0.1944 & 0 & 0.0006 & 0 & 0.0359 \\
\hline$T_{c l x}$ & 0.0004 & 0.0570 & 0.6058 & 0.8945 & 0.1912 & 0.2810 & 0.9483 & 0.5678 & 0.1954 & 0.0707 & 0.2231 \\
\hline
\end{tabular}

Table 11: P-values from the tests of the two covariance matrices of daily stock returns in the Information Technology sector.

\section{Appendix}

In this section, we prove Theorem 1 and Theorem 2 by using the same tools. To simplify the notation, we use $c_{1}, c_{2}, y_{1}, y_{2}, h$, and $\alpha$ instead of $c_{n_{1}}, c_{n_{2}}, y_{n_{1}}, y_{n_{2}}, h_{n}$, and $\alpha_{n}$, respectively. We calculate $l_{n}, \mu_{n}$ and $\nu_{n}$ in Theorem 1 and Theorem 2] based on Theorem 1.1 and Theorem 1.6 from Bai et al. [5], included here for convenience.

Lemma 3 (Theorem 1.1 in Bai et al. [5]). Under assumptions (1)-(4), the empirical spectral distribution (ESD) of the random Beta matrix $\mathbf{B}_{n}^{x}\left(\mathbf{X}_{1}, \mathbf{X} 2\right)$ with probability 1 weakly converges to a non-random distribution $\mathbf{F}^{\mathbf{B}}(x)$ whose density function is distributed as

$$
\begin{cases}\frac{(\alpha+1) \sqrt{\left(x_{r}-x\right)\left(x-x_{l}\right)}}{2 \pi y_{1} x(1-x)} & \text { when } x_{l}<x<x_{r} \\ 0 & \text { otherwise, }\end{cases}
$$

where $x_{l}, x_{r}=\frac{y_{2}\left(h \mp y_{1}\right)^{2}}{\left(y_{1}+y_{2}\right)^{2}}$.

Lemma 4 (Theorem 1.6 in Bai et al. [5]). In addition to conditions (1)-(4), we further assume that $f_{1}, \ldots . . . f_{k}$ are analytic functions on an open region containing the interval $\left[c_{l e}, c_{r i}\right]$, where $c_{l e}=v^{-1}\left(1-\sqrt{y_{1}}\right)^{2}$, ${ }_{165} c_{r i}=1-\alpha v^{-1}\left(1-\sqrt{y_{2}}\right)^{2}$, and $v$ is defined as $v=\left(1+\frac{y_{1}}{y_{2}}\right)\left(1-\sqrt{\frac{y_{1} y_{2}}{y_{1}+y_{2}}}\right)^{2}$.

Then, as $\min \left(n_{1}, n_{2}, p\right) \rightarrow \infty$, the random vector

$$
\left(\int f_{\gamma} \mathrm{d} \mathbf{G}_{\mathbf{n}}(x)\right), \quad \gamma=1, \ldots \ldots, k,
$$

where $\mathbf{G}_{\mathbf{n}}(x)=p\left(\mathbf{F}_{n}^{\mathbf{B}_{n}^{x}\left(\mathbf{X}_{1}, \mathbf{X}_{2}\right)}(x)-\mathbf{F}^{\mathbf{B}}(x)\right)$ converges weakly to a Gaussian vector $\left(\mathbf{G}_{f_{1}}, \ldots, \mathbf{G}_{f_{k}}\right)$ with the mean function

$$
\begin{gathered}
\boldsymbol{E G}_{f_{\gamma}=\frac{1}{4 \pi i}} \oint f_{\gamma}\left(\frac{z}{\alpha+z}\right) \mathrm{d} \log \left(\frac{\left(1-y_{2}\right) m_{3}^{2}(z)+2 m_{3}(z)+1-y_{1}}{\left(1+m_{3}(z)\right)^{2}}\right) \\
+\frac{\Delta_{1}}{2 \pi i} \oint y_{1} f_{\gamma}\left(\frac{z}{\alpha+z}\right)\left(1+m_{3}\right)^{-3} \mathrm{~d} m_{3}(z)
\end{gathered}
$$




$$
+\frac{\Delta_{2}}{4 \pi i} \oint f_{\gamma}\left(\frac{z}{\alpha+z}\right)\left(1-y_{2} m_{3}^{2}(z)\left(1+m_{3}(z)\right)^{-2}\right) \mathrm{d} \log \left(1-y_{2} m_{3}^{2}(z)\left(1+m_{3}(z)\right)^{-2}\right),
$$

and the covariance function

$$
\begin{gathered}
\operatorname{Cov}\left(\mathbf{G}_{f_{\gamma}}, \mathbf{G}_{\left.f_{\gamma^{\prime}}\right)=-\frac{1}{2 \pi^{2}}} \oint \oint \frac{f_{\gamma}\left(\frac{z_{1}}{\alpha+z_{1}}\right) f_{\gamma^{\prime}}\left(\frac{z_{2}}{\alpha+z_{2}}\right) \mathrm{d} m_{3}\left(z_{1}\right) \mathrm{d} m_{3}\left(z_{2}\right)}{\left(m_{3}\left(z_{1}\right)-m_{3}\left(z_{2}\right)\right)^{2}}\right. \\
-\frac{y_{1} \Delta_{1}+y_{2} \Delta_{2}}{4 \pi^{2}} \oint \oint \frac{f_{\gamma}\left(\frac{z_{1}}{\alpha+z_{1}}\right) f_{\gamma^{\prime}}\left(\frac{z_{2}}{\alpha+z_{2}}\right) \mathrm{d} m_{3}\left(z_{1}\right) \mathrm{d} m_{3}\left(z_{2}\right)}{\left(m_{3}\left(z_{1}\right)+1\right)^{2}\left(m_{3}\left(z_{2}\right)+1\right)^{2}},
\end{gathered}
$$

where

$$
\begin{aligned}
m_{0}(z) & =\frac{\left(1+y_{1}\right)(1-z)-\alpha z\left(1-y_{2}\right)+\sqrt{\left(\left(1-y_{1}\right)(1-z)+\alpha z\left(1-y_{2}\right)\right)^{2}-4 \alpha z(1-z)}}{2 z(1-z)\left(y_{1}(1-z)+\alpha z y_{2}\right)}-\frac{1}{z}, \\
m_{1}(z) & =\frac{\alpha}{(\alpha+z)^{2}} m_{0}\left(\frac{z}{\alpha+z}\right)-\frac{1}{\alpha+z}, \quad m_{2}(z)=-z^{-1}\left(1-y_{1}\right)+y_{1} m_{1}(z), \\
m_{m p}^{y_{2}}(z) & =\frac{1-y_{2}-z+\sqrt{\left(z-1-y_{2}\right)^{2}-4 y_{2}}}{2 y_{2} z}, \quad m_{3}(z)=y_{2} m_{m p}^{y_{2}}\left(-m_{2}(z)\right)+\left(m_{2}(z)\right)^{-1}\left(1-y_{2}\right) .
\end{aligned}
$$

The above contour integrals can be evaluated on any contour enclosing the interval $\left[\frac{\alpha c_{l e}}{1-c_{l e}}, \frac{\alpha c_{r i}}{1-c_{r i}}\right]:$ here, $i$ represents an imaginary unit.

Now, we are in position to prove Theorem 1

PROOF of the limit part $l_{n}$ in Theorem 1 To calculate the limit part

$$
p \int x \frac{(1+\alpha) \sqrt{\left(x_{r}-x\right)\left(x-x_{l}\right)}}{2 \pi y_{1} x(1-x)} \mathrm{d} x
$$

we first perform the transformations $x=\frac{y_{2}\left|y_{1}+h \xi\right|^{2}}{\left(y_{1}+y_{2}\right)^{2}}$ and $1-x=\frac{y_{1}\left|y_{2}-h \xi\right|^{2}}{\left(y_{1}+y_{2}\right)^{2}}$. Because $x$ appears in the molecular orbital of the integrand function, no residue is related to $y_{1}$. We assume that $y_{1}>1$. Clearly, as $x$ moves from $\frac{y_{2}\left(h-y_{1}\right)^{2}}{\left(y_{1}+y_{2}\right)^{2}}$ to $\frac{y_{2}\left(h+y_{1}\right)^{2}}{\left(y_{1}+y_{2}\right)^{2}}$ two times, $\xi$ runs along the unit circle in the positive direction. Thus, integral (8) is equivalent to

$$
p \frac{h^{2} i}{4 \pi\left(y_{1}+y_{2}\right)} \oint \frac{\left(\xi^{2}-1\right)^{2}}{\xi^{2}\left(y_{2}-h \xi\right)\left(\xi-\frac{h}{y_{2}}\right)} \mathrm{d} \xi
$$

According to the residue theorem, we obtain two poles $\left\{0, \frac{h}{y_{2}}\right\}$ in the unit disc when $y_{2}>1$, and the residues are

$$
-\frac{y_{2}^{2}+h^{2}}{y_{2} h^{2}}, \quad \frac{y_{2}^{2}-h^{2}}{y_{2} h^{2}} .
$$

Then, under the assumption $y_{2}>1,8$ yields

$$
p \frac{h^{2} i}{4 \pi\left(y_{1}+y_{2}\right)} \cdot 2 \pi i \cdot\left(-\frac{y_{2}^{2}+h^{2}}{y_{2} h^{2}}+\frac{y_{2}^{2}-h^{2}}{y_{2} h^{2}}\right)=p \frac{h^{2}}{\left(y_{1}+y_{2}\right) y_{2}} .
$$

In the same way, we obtain two poles $\left\{0, \frac{y_{2}}{h}\right\}$ and two residues

$$
-\frac{y_{2}^{2}+h^{2}}{y_{2}^{2} h}, \quad-\frac{y_{2}^{2}-h^{2}}{y_{2}^{2} h}
$$


under the assumption $y_{2}<1$. Then, we can calculate that $(8)$ is

$$
-p \frac{h y_{2} i}{4 \pi\left(y_{1}+y_{2}\right)} \cdot 2 \pi i \cdot\left(\frac{y_{2}^{2}+h^{2}}{y_{2}^{2} h}+\frac{y_{2}^{2}-h^{2}}{y_{2}^{2} h}\right)=p \frac{y_{2}}{y_{1}+y_{2}},
$$

which completes the proof.

PROOF of the mean part $\mu_{n}$ in Theorem 1 Because $m_{3}$ satisfies the equation

$$
z=-\frac{m_{3}(z)\left(m_{3}(z)+1-y_{1}\right)}{\left(1-y_{2}\right)\left(m_{3}(z)+\frac{1}{1-y_{2}}\right)}
$$

we perform an integral conversion $z=(1+h r \xi)\left(1+\frac{h}{r \xi}\right) /\left(1-y_{2}\right)^{2}$, where $r$ is a number greater than but close to 1 . For the same reason, we assume $y_{1}>1$ without loss of generality. The pole related to $y_{2}$ of the integrand is $\frac{h}{y_{2}}$ when $y_{2}>1$. The integral value is not changed by the transformation $\xi=\frac{1}{\xi}$; however, the residue point in the unit disc becomes $\frac{y_{2}}{h}$, which is the residue point under the assumption $y_{2}<1$. Therefore, we can assume that $y_{2}>1$. By solving the equation

$$
\frac{(1+h r \xi)\left(1+\frac{h}{r \xi}\right)}{\left(1-y_{2}\right)^{2}}=-\frac{m_{3}\left(m_{3}+1-y_{1}\right)}{\left(1-y_{2}\right) m_{3}+1}
$$

we obtain $m_{3}=-(1+h r \xi) /\left(1-y_{2}\right)$ or $m_{3}=-\left(1+\frac{h}{r \xi}\right) /\left(1-y_{2}\right)$. When $z$ runs in the positive direction along the unit circle around the support of $\mathbf{F}^{\mathbf{B}}(x), m_{3}$ runs in the opposite direction. Therefore, when $y_{2}>1$, we choose the outcome $m_{3}=-\left(1+\frac{h}{r \xi}\right) /\left(1-y_{2}\right)$. Based on the above discussion, we have

$$
\frac{z}{\alpha+z}=\frac{y_{2}|1+h r \xi|^{2}}{\left|y_{2}+h r \xi\right|^{2}}
$$

Therefore, we obtain the mean part

$$
\begin{aligned}
& \text { (3) }=\lim _{r \downarrow 1} \frac{1}{4 \pi i} \oint_{|\xi|=1} \frac{y_{2}(1+h r \xi)\left(1+\frac{h}{r \xi}\right)}{\left(y_{2}+h r \xi\right)\left(y_{2}+\frac{h}{r \xi}\right)} \cdot\left(\frac{1}{\xi-\frac{1}{r}}+\frac{1}{\xi+\frac{1}{r}}-\frac{2}{\xi+\frac{h}{y_{2} r}}\right) \mathrm{d} \xi, \\
& \text { (4) }=-\lim _{r \downarrow 1} \frac{\Delta_{1}}{2 \pi i} \oint y_{1} \frac{y_{2}(1+h r \xi)\left(1+\frac{h}{r \xi}\right)}{\left(y_{2}+h r \xi\right)\left(y_{2}+\frac{h}{r \xi}\right)} \cdot \frac{\left(1-y_{2}\right)^{2} h}{y_{2}^{3}} \cdot \frac{\xi}{\left(\xi+\frac{h}{y_{2} r}\right)^{3}} \mathrm{~d} \xi,
\end{aligned}
$$

$$
\text { (5) }=\lim _{r \downarrow 1} \frac{\Delta_{2}}{4 \pi i} \oint \frac{y_{2}(1+h r \xi)\left(1+\frac{h}{r \xi}\right)}{\left(y_{2}+h r \xi\right)\left(y_{2}+\frac{h}{r \xi}\right)} \cdot \frac{\left(y_{2}-1\right)\left(\xi^{2}-\frac{h^{2}}{y_{2} r^{2}}\right)}{y_{2}\left(\xi+\frac{h}{y_{2} r}\right)^{2}} \cdot\left(\frac{2 \xi}{\xi^{2}-\frac{h^{2}}{y_{2} r^{2}}}-\frac{2}{\xi+\frac{h}{y_{2} r}}\right) \mathrm{d} \xi .
$$

Based on the residue theorem, (3) has three poles,

$$
\frac{1}{r}, \quad-\frac{1}{r}, \quad-\frac{h}{y_{2} r}
$$

and three residues,

$$
\frac{y_{2}(1+h)^{2}}{\left(y_{2}+h\right)^{2}}, \quad \frac{y_{2}(1-h)^{2}}{\left(y_{2}-h\right)^{2}}, \quad \frac{2 h^{2} y_{1}}{\left(y_{1}+y_{2}\right)^{2}}
$$

Then,

$$
\text { (3) }=0 \text {. }
$$


Similarly,

$$
\text { (4) }=-\Delta_{1} \frac{y_{1}^{2} y_{2}^{2} h^{2}}{\left(y_{1}+y_{2}\right)^{4}} \text { and }(5)=-\Delta_{2} \frac{y_{1}^{2} y_{2}^{2} h^{2}}{\left(y_{1}+y_{2}\right)^{4}}
$$

Finally, we obtain the result of the mean part

$$
\mu_{n}=-\frac{\Delta_{1} y_{1}^{2} y_{2}^{2} h^{2}}{\left(y_{1}+y_{2}\right)^{4}}-\frac{\Delta_{2} y_{1}^{2} y_{2}^{2} h^{2}}{\left(y_{1}+y_{2}\right)^{4}}
$$

which completes the proof.

PROOF of the variance part in Theorem 1 . To calculate the variance part (6) and (7), we make analogous integral conversions

$$
z_{1}=\left(1+h r_{1} \xi_{1}\right)\left(1+\frac{h}{r_{1} \xi_{1}}\right) /\left(1-y_{2}\right)^{2}
$$

and

$$
z_{2}=\left(1+h r_{2} \xi_{2}\right)\left(1+\frac{h}{r_{2} \xi_{2}}\right) /\left(1-y_{2}\right)^{2} .
$$

Therefore, the relationship between $\xi_{l}$ and $m_{3}\left(z_{l}\right)$, where $l=1,2$, is as follows

$$
m_{3}\left(z_{1}\right)=-\frac{1+\frac{h}{r_{1} \xi_{1}}}{\left(1-y_{2}\right)} \text { and } m_{3}\left(z_{2}\right)=-\frac{1+\frac{h}{r_{2} \xi_{2}}}{\left(1-y_{2}\right)} .
$$

We assume that $r_{1}<r_{2}$ without loss of generality. When $y_{1}>1$ and $y_{2}>1$, according to the residue theorem, we obtain

$$
\begin{gathered}
\text { 6) }=2 \lim _{r_{2} \downarrow 1} \oint \frac{1}{2 \pi i} \cdot\left[\frac{y_{2}\left(1+h r_{2} \xi_{2}\right)\left(1+\frac{h}{r_{2} \xi_{2}}\right)}{\left(y_{2}+h r_{2} \xi_{2}\right)\left(y_{2}+\frac{h}{r_{2} \xi_{2}}\right)}\right] \\
\cdot\left\{\lim _{r_{1} \downarrow 1} \oint \frac{1}{2 \pi i} \cdot\left[\frac{y_{2}\left(1+h r_{1} \xi_{1}\right)\left(1+\frac{h}{r_{1} \xi_{1}}\right)}{\left(y_{2}+h r_{1} \xi_{1}\right)\left(y_{2}+\frac{h}{r_{1} \xi_{1}}\right)}\right] \cdot \frac{r_{1}}{\left(r_{1} \xi_{1}-r_{2} \xi_{2}\right)^{2}} \mathrm{~d} \xi_{1}\right\} \cdot r_{2} \mathrm{~d} \xi_{2} .
\end{gathered}
$$

Only one pole $-\frac{h}{y_{2}}$ exists in the unit disc for $\xi_{1}$, and the respective residue point is

$$
\frac{y_{1} y_{2} h\left(y_{2}-1\right)}{\left(y_{1}+y_{2}\right)\left(h+y_{2} \xi_{2}\right)^{2}}
$$

Then, we obtain the formula

$$
\text { (6) }=2 \lim _{r_{2} \downarrow 1} \oint \frac{1}{2 \pi i} \cdot\left[\frac{y_{2}\left(1+h r_{2} \xi_{2}\right)\left(1+\frac{h}{r_{2} \xi_{2}}\right)}{\left(y_{2}+h r_{2} \xi_{2}\right)\left(y_{2}+\frac{h}{r_{2} \xi_{2}}\right)}\right] \cdot \frac{y_{1}\left(y_{2}-1\right) h}{r_{2}^{2} y_{2}\left(y_{1}+y_{2}\right)} \cdot \frac{1}{\left(\xi_{2}+\frac{h}{y_{2} r_{2}}\right)^{2}} r_{2} \mathrm{~d} \xi_{2},
$$

which has only one pole, $-\frac{y_{2}}{r_{2} \xi_{2}}$, in the unit disc. Then, we have

$$
\text { (6) }=\frac{2 y_{1}^{2} y_{2}^{2} h^{2}}{\left(y_{1}+y_{2}\right)^{4}} \text {. }
$$

In the same way, we have the following calculation

$$
\text { (7) }=\left(y_{1} \Delta_{1}+y_{2} \Delta_{2}\right) \cdot\left\{\lim _{r_{1} \downarrow 1} \oint \frac{1}{2 \pi i} \cdot\left[\frac{y_{2}\left(1+h r_{1} \xi_{1}\right)\left(1+\frac{h}{r_{1} \xi_{1}}\right)}{\left(y_{2}+h r_{1} \xi_{1}\right)\left(y_{2}+\frac{h}{r_{1} \xi_{1}}\right)}\right] \cdot \frac{\left(1-y_{2}\right) r_{1} h \mathrm{~d} \xi_{1}}{\left(y_{2} r_{1} \xi_{1}+h\right)^{2}}\right\}
$$




$$
\begin{gathered}
\cdot\left\{\lim _{r_{2} \downarrow 1} \oint \frac{1}{2 \pi i} \cdot\left[\frac{y_{2}\left(1+h r_{2} \xi_{2}\right)\left(1+\frac{h}{r_{2} \xi_{2}}\right)}{\left(y_{2}+h r_{2} \xi_{2}\right)\left(y_{2}+\frac{h}{r_{2} \xi_{2}}\right)}\right] \cdot \frac{\left(1-y_{2}\right) r_{2} h \mathrm{~d} \xi_{2}}{\left(y_{2} r_{2} \xi_{2}+h\right)^{2}}\right\} \\
=\left(y_{1} \Delta_{1}+y_{2} \Delta_{2}\right) \cdot \frac{y_{1}^{2} y_{2}^{2} h^{4}}{\left(y_{1}+y_{2}\right)^{6}} .
\end{gathered}
$$

Therefore, we can conclude that

$$
\nu_{n}^{2}=\frac{2 y_{1}^{2} y_{2}^{2} h^{2}}{\left(y_{1}+y_{2}\right)^{4}}+\frac{\left(y_{1} \Delta_{1}+y_{2} \Delta_{2}\right) y_{1}^{2} y_{2}^{2} h^{4}}{\left(y_{1}+y_{2}\right)^{6}},
$$

which completes the proof.

We now give the proof for the statistic $T_{2}$.

PROOF of the limit part $\widetilde{l}_{n}$ in Theorem 2 To calculate the limit part $\widetilde{p l}_{n}$,

$$
p \int\left[c_{1}\left(\frac{x}{c_{1}}-1\right)^{2}+c_{2}\left(\frac{1-x}{c_{2}}-1\right)^{2}\right] \cdot \frac{(1+\alpha)}{\sqrt{\left(x_{r}-x\right)\left(x-x_{l}\right)}} 2 \pi y_{1} x(1-x) \mathrm{d} x,
$$

where

$$
x_{l}, x_{r}=\frac{y_{2}\left(h \mp y_{1}\right)^{2}}{\left(y_{1}+y_{2}\right)^{2}} .
$$

Performing the transformation

$$
x=\frac{y_{2}\left|y_{1}+h \xi\right|^{2}}{\left(y_{1}+y_{2}\right)^{2}}
$$

we have

$$
1-x=\frac{y_{1}\left|y_{2}-h \xi\right|^{2}}{\left(y_{1}+y_{2}\right)^{2}} .
$$

Thus,

$$
\begin{gathered}
p \int c_{1}\left(\frac{x}{c_{1}}-1\right)^{2} \cdot \frac{(1+\alpha) \sqrt{\left(x_{r}-x\right)\left(x-x_{l}\right)}}{2 \pi y_{1} x(1-x)} \mathrm{d} x \\
=\frac{p h^{2} i}{4 \pi} \oint y_{2}\left[\frac{\left|y_{1}+h \xi\right|^{2}}{\left(y_{1}+y_{2}\right)}-1\right]^{2} \cdot \frac{\left(\xi^{2}-1\right)^{2}}{\xi^{3}\left|y_{1}+h \xi\right|^{2}\left|y_{2}-h \xi\right|^{2}} \mathrm{~d} \xi \\
=\frac{p h^{2} i}{4 \pi} \oint y_{2} \frac{\left|y_{1}+h \xi\right|^{2}}{\left(y_{1}+y_{2}\right)^{2}} \cdot \frac{\left(\xi^{2}-1\right)^{2}}{\xi^{3}\left|y_{2}-h \xi\right|^{2}} \mathrm{~d} \xi \\
+\frac{p h^{2} i}{4 \pi} \oint y_{2} \frac{-2}{\left(y_{1}+y_{2}\right)} \cdot \frac{\left(\xi^{2}-1\right)^{2}}{\xi^{3}\left|y_{2}-h \xi\right|^{2}} \mathrm{~d} \xi \\
+\frac{p h^{2} i}{4 \pi} \oint y_{2} \frac{\left(\xi^{2}-1\right)^{2}}{\xi^{3}\left|y_{1}+h \xi\right|^{2}\left|y_{2}-h \xi\right|^{2}} \mathrm{~d} \xi .
\end{gathered}
$$

Under the assumptions $y_{1}>1$ and $y_{2}>1$, two poles exist inside the unit circle in (11)

$0, \frac{h}{y_{2}}$, 
and the respective residues are

$$
\frac{y_{2}}{\left(y_{1}+y_{2}\right)^{2}} \cdot\left(\frac{2 y_{1}}{y_{2}}-\frac{\left(h^{2}+y_{2}^{2}\right)\left(y_{1}+y_{2}\right)^{2}}{h^{2} y_{2}^{3}}\right), \quad \frac{y_{2}}{\left(y_{1}+y_{2}\right)^{2}} \cdot \frac{\left(y_{1}+y_{2}\right)^{3}\left(y_{2}-1\right)}{y_{2}^{3} h^{2}} .
$$

Applying the residue theorem to [12], there are two poles

$$
0, \quad \frac{h}{y_{2}}
$$

and two residues

$$
\frac{-2 y_{2}}{\left(y_{1}+y_{2}\right)} \cdot \frac{-\left(h^{2}+y_{2}^{2}\right)}{y_{2}^{2} h^{2}}, \quad \frac{-2 y_{2}}{\left(y_{1}+y_{2}\right)} \cdot \frac{y_{2}^{2}-h^{2}}{y_{2}^{2} h^{2}} .
$$

Following the same method, we obtain three poles

$$
0, \quad-\frac{h}{y_{2}}, \quad \frac{h}{y_{2}}
$$

and three residues

$$
-y_{2} \cdot \frac{1}{y_{1} y_{2} h^{2}}, \quad \frac{1}{y_{1}} \cdot \frac{\left(y_{1}-1\right) y_{2}}{\left(y_{1}+y_{2}\right) h^{2}}, \quad \frac{1}{y_{1}} \cdot \frac{\left(y_{2}-1\right) y_{1}}{\left(y_{1}+y_{2}\right) h^{2}}
$$

in (13). Then, we have

$$
[10]=c_{1} p h^{2}\left[\frac{y_{1}}{y_{2}^{3}}+\frac{1}{y_{1} y_{2}}-\frac{1}{y_{2}^{2}}-\frac{y_{1}}{y_{2}\left(y_{1}+y_{2}\right)}\right] .
$$

In the same way,

$$
p \int c_{2}\left(\frac{1-x}{c_{2}}-1\right)^{2} \cdot \frac{(1+\alpha) \sqrt{\left(x_{r}-x\right)\left(x-x_{l}\right)}}{2 \pi y_{1} x(1-x)} \mathrm{d} x
$$

$$
\begin{gathered}
=\frac{p h^{2} i}{4 \pi} \oint y_{1}\left[\frac{\left|y_{2}-h \xi\right|^{2}}{\left(y_{1}+y_{2}\right)}-1\right]^{2} \cdot \frac{\left(\xi^{2}-1\right)^{2}}{\xi^{3}\left|y_{1}+h \xi\right|^{2}\left|y_{2}-h \xi\right|^{2}} \mathrm{~d} \xi \\
=c_{2} p h^{2}\left[\frac{y_{2}}{y_{1}^{3}}+\frac{1}{y_{1} y_{2}}-\frac{1}{y_{1}^{2}}-\frac{y_{2}}{y_{1}\left(y_{1}+y_{2}\right)}\right] .
\end{gathered}
$$

Thus,

$$
\text { (9) }=p h^{2}\left[\frac{1}{y_{1}^{2}}+\frac{1}{y_{2}^{2}}-\frac{1}{y_{1}+y_{2}}-\frac{1}{y_{1} y_{2}}\right] \text {. }
$$

When the assumptions are $y_{1}>1$ and $y_{2}<1$, we use the same transformation

$$
x=\frac{y_{2}\left|y_{1}+h \xi\right|}{\left(y_{1}+y_{2}\right)^{2}} .
$$

In this case, the poles of (10) and (14) are

$$
0, \quad-\frac{h}{y_{1}}, \quad \frac{y_{2}}{h} .
$$

Therefore,

$$
(10)=\frac{p h^{2} i}{4 \pi} \oint y_{2}\left[\frac{\left|y_{1}+h \xi\right|^{2}}{\left(y_{1}+y_{2}\right)}-1\right]^{2} \cdot \frac{\left(\xi^{2}-1\right)^{2}}{\xi^{3}\left|y_{1}+h \xi\right|^{2}\left|y_{2}-h \xi\right|^{2}} \mathrm{~d} \xi
$$




$$
\begin{gathered}
=\frac{p h^{2} i}{4 \pi} \oint y_{2} \frac{\left|y_{1}+h \xi\right|^{2}}{\left(y_{1}+y_{2}\right)^{2}} \cdot \frac{\left(\xi^{2}-1\right)^{2}}{\xi^{3}\left|y_{2}-h \xi\right|^{2}} \mathrm{~d} \xi \\
+\frac{p h^{2} i}{4 \pi} \oint y_{2} \frac{-2}{\left(y_{1}+y_{2}\right)} \cdot \frac{\left(\xi^{2}-1\right)^{2}}{\xi^{3}\left|y_{2}-h \xi\right|^{2}} \mathrm{~d} \xi \\
+\frac{p h^{2} i}{4 \pi} \oint y_{2} \cdot \frac{\left(\xi^{2}-1\right)^{2}}{\xi^{3}\left|y_{1}+h \xi\right|^{2}\left|y_{2}-h \xi\right|^{2}} \mathrm{~d} \xi \\
=\frac{p h^{2} i}{4 \pi} \cdot 2 \pi i \cdot \frac{1}{\left(y_{1}+y_{2}\right)^{2}} \cdot\left[\frac{\left(y_{1}+y_{2}\right)^{3}\left(1-y_{2}\right)}{y_{2}^{2} h^{2}}+2 y_{1}-\frac{\left(h^{2}+y_{2}^{2}\right)\left(y_{1}+y_{2}\right)^{2}}{h^{2} y_{2}^{2}}\right] \\
+\frac{p h^{2} i}{4 \pi} \cdot 2 \pi i \cdot \frac{2 y_{2}}{h\left(y_{1}+y_{2}\right)} \cdot\left[\frac{\left(y_{1}+y_{2}\right)\left(y_{2}-1\right)}{y_{2}^{2} h}+\frac{\left(h^{2}+y_{2}^{2}\right)}{y_{2}^{2} h}\right]
\end{gathered}
$$

205

$$
+\frac{p h^{2} i}{4 \pi} \cdot 2 \pi i \cdot \frac{-y_{2}}{y_{1} h} \cdot\left[\frac{\left(y_{2}-1\right) y_{1}}{\left(y_{1}+y_{2}\right) h y_{2}}+\frac{\left(1-y_{1}\right)}{\left(y_{1}+y_{2}\right) h}+\frac{1}{h y_{2}}\right]
$$

and

$$
\begin{gathered}
\sqrt{14})=\frac{p h^{2} i}{4 \pi} \oint y_{1}\left[\frac{\left|y_{2}-h \xi\right|^{2}}{\left(y_{1}+y_{2}\right)}-1\right]^{2} \cdot \frac{\left(\xi^{2}-1\right)^{2}}{\xi^{3}\left|y_{1}+h \xi\right|^{2}\left|y_{2}-h \xi\right|^{2}} \mathrm{~d} \xi \\
=\frac{p h^{2} i}{4 \pi} \cdot 2 \pi i \cdot \frac{1}{\left(y_{1}+y_{2}\right)^{2}} \cdot\left[\frac{\left(y_{1}+y_{2}\right)^{3}\left(y_{1}-1\right)}{y_{1}^{2} h^{2}}+2 y_{2}-\frac{\left(h^{2}+y_{1}^{2}\right)\left(y_{1}+y_{2}\right)^{2}}{h^{2} y_{1}^{2}}\right] \\
+\frac{p h^{2} i}{4 \pi} \cdot 2 \pi i \cdot \frac{-2}{\left(y_{1}+y_{2}\right)} \cdot\left[\frac{\left(y_{1}+y_{2}\right)\left(y_{1}-1\right)}{y_{1} h^{2}}+\frac{-\left(h^{2}+y_{1}^{2}\right)}{y_{1} h^{2}}\right] \\
+\frac{p h^{2} i}{4 \pi} \cdot 2 \pi i \cdot \frac{1}{y_{2}} \cdot\left[\frac{\left(1-y_{2}\right) y_{1}}{\left(y_{1}+y_{2}\right) h^{2}}+\frac{\left(y_{1}-1\right) y_{2}}{\left(y_{1}+y_{2}\right) h^{2}}+\frac{-1}{h^{2}}\right] .
\end{gathered}
$$

Thus, using the residue theorem, we can conclude that

$$
\text { (9) }=p h^{2}\left[\frac{1}{y_{1}^{2}}+\frac{1}{y_{2} h^{2}}-\frac{1}{y_{1}+y_{2}}-\frac{1}{y_{1} y_{2}}\right]
$$

under the conditions $y_{1}>1$ and $y_{2}<1$.

When $y_{1}<1$ and $y_{2}<1$, we have three poles

$$
0, \quad-\frac{y_{1}}{h}, \quad \frac{y_{2}}{h}
$$

Thus,

$$
\text { (10) }=\frac{p h^{2} i}{4 \pi} \cdot 2 \pi i \cdot \frac{1}{\left(y_{1}+y_{2}\right)^{2}} \cdot\left[\frac{\left(y_{1}+y_{2}\right)^{3}\left(1-y_{2}\right)}{h^{2} y_{2}^{2}}+2 y_{1}-\frac{\left(h^{2}+y_{2}^{2}\right)\left(y_{1}+y_{2}\right)^{2}}{h^{2} y_{2}^{2}}\right]
$$


215

$$
\begin{aligned}
& +\frac{p h^{2} i}{4 \pi} \cdot 2 \pi i \cdot \frac{2 y_{2}}{h\left(y_{1}+y_{2}\right)} \cdot\left[\frac{\left(y_{1}+y_{2}\right)\left(y_{2}-1\right)}{y_{2}^{2} h}+\frac{\left(h^{2}+y_{2}^{2}\right)}{y_{2}^{2} h}\right] \\
& +\frac{p h^{2} i}{4 \pi} \cdot 2 \pi i \cdot \frac{-y_{2}}{y_{1} h} \cdot\left[\frac{\left(y_{2}-1\right) y_{1}}{\left(y_{1}+y_{2}\right) h y_{2}}+\frac{\left(y_{1}-1\right)}{\left(y_{1}+y_{2}\right) h}+\frac{1}{h y_{2}}\right]
\end{aligned}
$$

$$
\begin{gathered}
\text { (14) }=\frac{p h^{2} i}{4 \pi} \cdot 2 \pi i \cdot \frac{1}{\left(y_{1}+y_{2}\right)^{2}} \cdot\left[\frac{\left(y_{1}+y_{2}\right)^{3}\left(1-y_{1}\right)}{y_{1}^{2} h^{2}}+2 y_{2}-\frac{\left(h^{2}+y_{1}^{2}\right)\left(y_{1}+y_{2}\right)^{2}}{h^{2} y_{1}^{2}}\right] \\
+\frac{p h^{2} i}{4 \pi} \cdot 2 \pi i \cdot \frac{-2}{\left(y_{1}+y_{2}\right)} \cdot\left[\frac{\left(y_{1}+y_{2}\right)\left(1-y_{1}\right)}{y_{1} h^{2}}+\frac{-\left(h^{2}+y_{1}^{2}\right)}{y_{1} h^{2}}\right] \\
+\frac{p h^{2} i}{4 \pi} \cdot 2 \pi i \cdot \frac{1}{y_{2}} \cdot\left[\frac{\left(1-y_{2}\right) y_{1}}{\left(y_{1}+y_{2}\right) h^{2}}+\frac{\left(1-y_{1}\right) y_{2}}{\left(y_{1}+y_{2}\right) h^{2}}+\frac{-1}{h^{2}}\right] .
\end{gathered}
$$

Under these conditions,

$$
\text { (9) }=p h^{2}\left[\frac{1}{h^{2}}-\frac{1}{y_{1}+y_{2}}\right],
$$

we can obtain the conclusion for the case where $y_{1}<1$ and $y_{2}>1$ in a similar manner. According to the discussion above, the limit part is

$$
\tilde{l}_{n}=\frac{y_{n_{1}} y_{n_{2}}}{y_{n_{1}}+y_{n_{2}}}+\frac{\left(1-y_{n_{1}}\right) y_{n_{2}}}{y_{n_{1}}^{2}} \delta_{\left(y_{n_{1}}>1\right)}+\frac{y_{n_{1}}\left(1-y_{n_{2}}\right)}{y_{n_{2}}^{2}} \delta_{\left(y_{n_{2}}>1\right)}
$$

PROOF of the mean part $\tilde{\mu}_{n}$ in Theorem 2. According to the above discussion, we assume that $y_{1}>$ $1, y_{2}>1$. From the transformation

$$
m_{3}=-(1+h r \xi) /\left(1-y_{2}\right),
$$

and its relationship with $m_{3}, z$ satisfies

$$
z=-\frac{m_{3}\left(m_{3}+1-y_{1}\right)}{\left(1-y_{2}\right) m_{3}+1} .
$$

We then obtain

$$
\frac{z}{\alpha+z}=\frac{y_{2}|1+h r \xi|^{2}}{\left|y_{2}+h r \xi\right|^{2}} \text { and } 1-\frac{z}{\alpha+z}=\frac{y_{1}\left(y_{2}-1\right)^{2}}{\left|y_{2}+h r \xi\right|^{2}} .
$$

In this mean part, we calculate the integrand $f_{\gamma}\left(\frac{z}{\alpha+z}\right)$, which is equivalent to

$$
c_{1}\left(\frac{1}{c_{1}} \cdot \frac{z}{\alpha+z}-1\right)^{2}+c_{2}\left(\frac{1}{c_{2}} \cdot\left(1-\frac{z}{\alpha+z}\right)-1\right)^{2} .
$$

According to the above discussion,

$$
(15)=c_{1}\left(\frac{1}{c_{1}} \cdot \frac{y_{2}|1+h r \xi|^{2}}{\left|y_{2}+h r \xi\right|^{2}}-1\right)^{2}+c_{2}\left(\frac{1}{c_{2}} \cdot \frac{y_{1}\left(y_{2}-1\right)^{2}}{\left|y_{2}+h r \xi\right|^{2}}-1\right)^{2}
$$




$$
\begin{gathered}
=c_{1}\left(\frac{|1+h r \xi|^{2}\left(y_{1}+y_{2}\right)}{\left|y_{2}+h r \xi\right|^{2}}-1\right)^{2}+c_{2}\left(\frac{\left(y_{2}-1\right)^{2}\left(y_{1}+y_{2}\right)}{\left|y_{2}+h r \xi\right|^{2}}-1\right)^{2} \\
=\frac{y_{2}\left(|1+h r \xi|^{2}\left(y_{1}+y_{2}\right)-\left|y_{2}+h r \xi\right|^{2}\right)^{2}}{\left(y_{1}+y_{2}\right)\left|y_{2}+h r \xi\right|^{4}}+\frac{y_{1}\left(\left(y_{2}-1\right)^{2}\left(y_{1}+y_{2}\right)-\left|y_{2}+h r \xi\right|^{2}\right)^{2}}{\left(y_{1}+y_{2}\right)\left|y_{2}+h r \xi\right|^{4}} \\
=\frac{y_{1} y_{2}\left(y_{1}+y_{2}\right)\left(h^{2}-y_{2}^{2}+2 y_{2}+h r \xi+\frac{h}{r \xi}\right)^{2}}{\left(y_{1}+y_{2}\right)\left|y_{2}+h r \xi\right|^{4}}=\frac{y_{1} y_{2}\left(|1+h r \xi|^{2}-\left(y_{2}-1\right)^{2}\right)^{2}}{\left|y_{2}+h r \xi\right|^{4}}
\end{gathered}
$$

$$
\begin{gathered}
(3)+(4)+(5)=\lim _{r \downarrow 1} \frac{1}{4 \pi i} \oint_{|\xi|=1} \frac{y_{1} y_{2}\left[|1+h r \xi|^{2}-\left(y_{2}-1\right)^{2}\right]^{2}}{\left|y_{2}+h r \xi\right|^{4}} \cdot\left(\frac{1}{\xi-\frac{1}{r}}+\frac{1}{\xi+\frac{1}{r}}-\frac{2}{\xi+\frac{h}{y_{2} r}}\right) \mathrm{d} \xi \\
-\lim _{r \downarrow 1} \frac{\Delta_{1}}{2 \pi i} \oint y_{1} \frac{y_{1} y_{2}\left[|1+h r \xi|^{2}-\left(y_{2}-1\right)^{2}\right]^{2}}{\left|y_{2}+h r \xi\right|^{4}} \cdot \frac{\left(1-y_{2}\right)^{2} h}{y_{2}^{3}} \cdot \frac{\xi}{\left(\xi+\frac{h}{y_{2} r}\right)^{3}} \mathrm{~d} \xi \\
=\lim _{r \downarrow 1} \frac{\Delta_{2}}{4 \pi i} \oint \frac{y_{1} y_{2}\left[|1+h r \xi|^{2}-\left(y_{2}-1\right)^{2}\right]^{2}}{\left|y_{2}+h r \xi\right|^{4}} \cdot \frac{\left(y_{2}-1\right)\left(\xi^{2}-\frac{h^{2}}{y_{2} r^{2}}\right)}{y_{2}\left(\xi+\frac{h}{y_{2} r}\right)^{2}} \cdot\left[\frac{2 \xi}{\left(\xi^{2}-\frac{h^{2}}{y_{2} r^{2}}\right)}-\frac{2}{\xi+\frac{h}{y_{2} r}}\right] \mathrm{d} \xi .
\end{gathered}
$$

According to Cauchy's residue theorem, we have

$$
\tilde{\mu}_{n}=\frac{y_{1} y_{2} h^{2}}{\left(y_{1}+y_{2}\right)^{2}}+\Delta_{1} \frac{y_{1}^{2} y_{2} h^{2}\left(h^{2}+2 y_{2}\left(y_{2}-y_{1}\right)\right)}{\left(y_{1}+y_{2}\right)^{4}}+\Delta_{2} \frac{y_{2}^{2} y_{1} h^{2}\left(h^{2}+2 y_{1}\left(y_{1}-y_{2}\right)\right)}{\left(y_{1}+y_{2}\right)^{4}}
$$

which completes the proof of the mean part.

PROOF of the variance part in Theorem 2 Under the case where $y_{1}>1$ and $y_{2}>1$, using the transformation discussed above, we can easily find that

$$
\begin{gathered}
\frac{\mathrm{d} m_{3}\left(z_{1}\right) \mathrm{d} m_{3}\left(z_{2}\right)}{\left(m_{3}\left(z_{1}\right)-m_{3}\left(z_{2}\right)\right)^{2}}=\frac{\left(1-y_{2}\right)^{2}}{\left(\frac{h}{r_{1} \xi_{1}}-\frac{h}{r_{2} \xi_{2}}\right)^{2}} \cdot \frac{h \mathrm{~d} \xi_{1}}{\left(1-y_{2}\right) r_{1} \xi_{1}^{2}} \cdot \frac{h \mathrm{~d} \xi_{2}}{\left(1-y_{2}\right) r_{2} \xi_{2}^{2}}=\frac{r_{1} r_{2}}{\left(r_{1} \xi_{1}-r_{2} \xi_{2}\right)^{2}} \mathrm{~d} \xi_{1} \mathrm{~d} \xi_{2}, \\
\frac{\mathrm{d} m_{3}\left(z_{1}\right)}{\left(m_{3}\left(z_{1}\right)+1\right)^{2}}=\frac{\left(1-y_{2}\right)^{2}}{\left(y_{2}+\frac{h}{r_{1} \xi_{1}}\right)^{2}} \cdot \frac{h \mathrm{~d} \xi_{1}}{\left(1-y_{2}\right) r_{1} \xi_{1}^{2}}=\frac{\left(1-y_{2}\right) r_{1} h \mathrm{~d} \xi_{1}}{\left(y_{2} r_{1} \xi_{1}+h\right)^{2}}, \\
\frac{\mathrm{d} m_{3}\left(z_{2}\right)}{\left(m_{3}\left(z_{2}\right)+1\right)^{2}}=\frac{\left(1-y_{2}\right)^{2}}{\left(y_{2}+\frac{h}{r_{2} \xi_{2}}\right)^{2}} \cdot \frac{h \mathrm{~d} \xi_{2}}{\left(1-y_{2}\right) r_{2} \xi_{2}^{2}}=\frac{\left(1-y_{2}\right) r_{2} h \mathrm{~d} \xi_{2}}{\left(y_{2} r_{2} \xi_{2}+h\right)^{2}} .
\end{gathered}
$$

Thus,

$$
(6)+(7)=2 \lim _{r_{2} \downarrow 1} \oint \frac{1}{2 \pi i} \cdot \frac{y_{1} y_{2}\left[\left|1+h_{2} r_{2} \xi\right|^{2}-\left(y_{2}-1\right)^{2}\right]^{2}}{\left|y_{2}+h_{2} r_{2} \xi\right|^{4}}
$$

235

$$
\cdot\left\{\lim _{r_{1} \downarrow 1} \oint \frac{1}{2 \pi i} \cdot \frac{y_{1} y_{2}\left[\left|1+h_{1} r_{1} \xi\right|^{2}-\left(y_{2}-1\right)^{2}\right]^{2}}{\left|y_{2}+h_{1} r_{1} \xi\right|^{4}} \cdot \frac{r_{1}}{\left(r_{1} \xi_{1}-r_{2} \xi_{2}\right)^{2}} \mathrm{~d} \xi_{1}\right\} \cdot r_{2} \mathrm{~d} \xi_{2}
$$




$$
\begin{gathered}
+c\left\{\lim _{r_{1} \downarrow 1} \oint \frac{1}{2 \pi i} \cdot\left[\frac{y_{1} y_{2}\left[\left|1+h_{2} r_{2} \xi\right|^{2}-\left(y_{2}-1\right)^{2}\right]^{2}}{\left|y_{2}+h_{2} r_{2} \xi\right|^{4}}\right] \cdot \frac{\left(1-y_{2}\right) r_{1} h \mathrm{~d} \xi_{1}}{\left(y_{2} r_{1} \xi_{1}+h\right)^{2}}\right\} \\
\cdot\left\{\lim _{r_{2} \downarrow 1} \oint \frac{1}{2 \pi i} \cdot\left[\frac{y_{1} y_{2}\left[\left|1+h_{1} r_{1} \xi\right|^{2}-\left(y_{2}-1\right)^{2}\right]^{2}}{\left|y_{2}+h_{1} r_{1} \xi\right|^{4}}\right] \cdot \frac{\left(1-y_{2}\right) r_{2} h \mathrm{~d} \xi_{2}}{\left(y_{2} r_{2} \xi_{2}+h\right)^{2}}\right\},
\end{gathered}
$$

where $c=y_{1} \Delta_{1}+y_{2} \Delta_{2}$ for the sake of brevity. According to Cauchy's residue theorem,

$$
\tilde{\nu}_{n}^{2}=\frac{4 y_{1}^{2} y_{2}^{2} h^{2}\left(h^{2}+2\left(y_{1}-y_{2}\right)^{2}\right)}{\left(y_{1}+y_{2}\right)^{4}}+c \frac{4 y_{1}^{2} y_{2}^{2} h^{4}\left(y_{1}-y_{2}\right)^{2}}{\left(y_{1}+y_{2}\right)^{6}},
$$

which completes the proof.

\section{Acknowledgments}

The authors would like to thank the anonymous referee and associate editor for their invaluable and constructive comments. The research was partially supported by NSFC (No. 11571067, 11771073) and Foundation of Jilin Educational Committee (No. JJKH20190288KJ).

\section{References}

[1] Z. Bai and J. W. Silverstein. Clt for linear spectral statistics of large dimension sample covariance matrices. The Annals of Probability, 32(1A):553-605, 2004.

[2] Z. Bai and J. Yao. Central limit theorems for eigenvalues in a spiked population model. Annales de l'Institut Henri Poincaré, Probabilités et Statistiques, 44(3):447-474, 2008.

[3] Z. Bai, D. Jiang, J. Yao, and S. Zheng. Corrections to LRT on large-dimensional covariance matrix by RMT. Annals of Statistics, 37(6B):3822-3840, 2009.

[4] Z. Bai, D. Jiang, J. Yao, and S. Zheng. Testing linear hypotheses in high-dimensional regressions. Statistics, 47(6):1207-1223, 2013.

[5] Z. Bai, H. Jiang, G. Pan, and W. Zhou. Convergence of the empirical spectral distribution function of Beta matrices. Bernoulli, 21(3):1538-1574, 2015.

[6] J. Baik and J. W. Silverstein. Eigenvalues of large sample covariance matrices of spiked population models. Journal of multivariate analysis, 97(6):1382-1408, 2006.

[7] M. Birke and H. Dette. A note on testing the covariance matrix for large dimension. Statistics \& Probability Letters, 74(3):281-289, 2005.

[8] T. Cai and Z. Ma. Optimal hypothesis testing for high dimensional covariance matrices. Bernoulli, 19(5B):2359-2388, 2013. 
[9] T. Cai, W. Liu, and Y. Xia. Two-sample covariance matrix testing and support recovery in highdimensional and sparse settings. Journal of the American Statistical Association, 108(501):265-277, 2013.

[10] H. Chen and T. Jiang. A study of two high-dimensional likelihood ratio tests under alternative hypotheses. Random Matrices: Theory and Applications, 7(1):1750016, 2018.

[11] S. X. Chen, L. X. Zhang, and P. S. Zhong. Tests for high-dimensional covariance matrices. Journal of the American Statistical Association, 105(490):810-819, 2010.

[12] T. J. Fisher, X. Sun, and C. M. Gallagher. A new test for sphericity of the covariance matrix for high dimensional data. Journal of Multivariate Analysis, 101(10):2554-2570, 2010.

[13] C. Gao, C. D. Brown, and B. E. Engelhardt. A latent factor model with a mixture of sparse and dense factors to model gene expression data with confounding effects. arXiv:1310.4792, 2013.

[14] D. Jiang, T. Jiang, and F. Yang. Likelihood ratio tests for covariance matrices of high-dimensional normal distributions. Journal of Statistical Planning and Inference, 142(8):2241-2256, 2012.

[15] I. M. Johnstone. On the distribution of the largest principal component. The Annals of Statistics, 29 (3):295-327, 2000.

[16] O. Ledoit and M. Wolf. Some hypothsis tests for the covariance matrix when the dimension is large compared to the sample size. The Annals of Statistics, 30(4):1081-1102, 2002.

[17] J. Li and S. X. Chen. Two sample tests for high-dimensional covariance matrices. Annals of Statistics, 40(2):908-940, 2012.

[18] V. A. Marchenko and L. A. Pastur. Distribution of eigenvalues for some sets of random matrices. Mathematics of the USSR-Sbornik, 1(4):457-483, 1967.

[19] D. Passemier, M. R. McKay, and Y. Chen. Asymptotic linear spectral statistics for spiked hermitian random matrices. Journal of Statistical Physics, 160(1):120-150, 2015.

[20] D. Paul. Asymptotics of sample eigenstructure for a large dimensional spiked covariance model. Statistica Sinica, 17(4):1617-1642, 2007.

[21] K. C. S. Pillai. On some distribution problems in multivariate analysis. North Carolina State University. Dept. of Statistics, 1954.

[22] Y. Qiu and S. X. Chen. Test for bandedness of high-dimensional covariance matrices and bandwidth estimation. The Annals of Statistics, 40(3):1285-1314, 2012. 
[23] J. R. Schott. A high-dimensional test for the equality of the smallest eigenvalues of a covariance matrix. Journal of Multivariate Analysis, 97(4):827-843, 2006.

[24] J. R. Schott. A test for the equality of covariance matrices when the dimension is large relative to the sample sizes. Computational Statistics \& Data Analysis, 51(12):6535-6542, 2007.

[25] M. S. Srivastava. Some tests concerning the covariance matrix in high dimensional data. Journal of the Japan Statistical Society, 35(2):251-272, 2005.

295 [26] M. S. Srivastava. Multivariate theory for analyzing high dimensional data. Journal of the Japan Statistical Society, 37(1):53-86, 2007.

[27] M. S. Srivastava and H. Yanagihara. Testing the equality of several covariance matrices with fewer observations than the dimension. Journal of Multivariate Analysis, 101(6):1319-1329, 2010.

[28] M. S. Srivastava, T. Kollo, and D. V. Rosen. Some tests for the covariance matrix with fewer observations than the dimension under non-normality. Journal of Multivariate Analysis, 102(6):1090-1103, 2011.

[29] T.-L. Wu and P. Li. Tests for high-dimensional covariance matrices using random matrix projection. arXiv:1310.4792, 2015.

[30] C. Zhang, Z. Bai, J. Hu, and C. Wang. Multi-sample test for high-dimensional covariance matrices. Communication in Statistics- Theory and Methods, 47(13):3161-3177, 2018.

[31] Q. Zhang, J. Hu, and Z. Bai. Invariant test based on the modified correction to lrt for the equality of two high-dimensional covariance matrices. Electronic Journal of Statistics, 13(1):850-881, 2019.

[32] S. Zheng. Central limit theorems for linear spectral statistics of large dimensional F-matrices. Annales de l'institut Henri Poincare (B) Probability and Statistics, 48(2):444-476, 2012.

310 [33] S. Zheng, Z. Bai, and J. Yao. Substitution principle for clt of linear spectral statistics of highdimensional sample covariance matrices with applications to hypothesis testing. The Annals of Statistics, 43(2):546-591, 2015. 